\title{
Structural and magnetic properties of granular Co-Pt multilayers with perpendicular magnetic anisotropy
}

\author{
A. I. Figueroa, ${ }^{1, *}$ J. Bartolomé, ${ }^{1}$ L. M. García, ${ }^{1}$ F. Bartolomé, ${ }^{1}$ O. Bunău, ${ }^{1}$ J. Stankiewicz, ${ }^{1}$ L. Ruiz, ${ }^{2}$ J. M. González-Calbet, ${ }^{2}$ \\ F. Petroff, ${ }^{3}$ C. Deranlot, ${ }^{3}$ S. Pascarelli, ${ }^{4}$ P. Bencok, ${ }^{4}$ N. B. Brookes, ${ }^{4}$ F. Wilhelm, ${ }^{4}$ A. Smekhova, ${ }^{4}$ and A. Rogalev ${ }^{4}$ \\ ${ }^{1}$ Instituto de Ciencia de Materiales de Aragón (ICMA), CSIC - Universidad de Zaragoza. \\ Departamento de Física de la Materia Condensada, E-50009 Zaragoza, Spain \\ ${ }^{2}$ Departamento de Química Inorgánica, Universidad Complutense de Madrid, E-28040 Madrid, Spain \\ ${ }^{3}$ Unité Mixte de Physique CNRS/Thales, F-91767 Palaiseau Cedex, France and Université Paris-Sud, F-91405 Orsay Cedex, France \\ ${ }^{4}$ European Synchrotron Radiation Facility (ESRF), F-38043 Grenoble, France
}

(Received 23 September 2014; published 17 November 2014)

\begin{abstract}
We present a study of granular Co-Pt multilayers by means of high-resolution transmission electron microscopy (HRTEM), extended x-ray absorption fine structure (EXAFS), SQUID-based magnetic measurements, anomalous Hall effect (AHE), and x-ray magnetic circular dichroism (XMCD). We describe these granular films as composed of particles with a pure cobalt core surrounded by an alloyed Co-Pt interface, embedded in a Pt matrix. The alloy between the $\mathrm{Co}$ and $\mathrm{Pt}$ in these granular films, prepared by room temperature sputter deposition, results from interdiffusion of the atoms. The presence of this alloy gives rise to a high perpendicular magnetic anisotropy (PMA) in the granular films, as consequence of the anisotropy of the orbital moment in the Co atoms in the alloy, and comparable to that of highly-ordered CoPt $L 1_{0}$ alloy films. Their magnetic properties are those of ferromagnetically coupled particles, whose coupling is strongly temperature dependent: at low temperatures, the granular sample is ferromagnetic with a high coercive field; at intermediate temperatures the granular film behaves as an amorphous asperomagnet, with a coupling between the grains mediated by the polarized Pt, and at high temperatures, the sample has a superparamagnetic behavior. The coupling/decoupling between the grains in our Co-Pt granular films can be tailored by variation of the amount of $\mathrm{Pt}$ in the samples.
\end{abstract}

DOI: 10.1103/PhysRevB.90.174421

PACS number(s): 75.75.Fk, 75.30.Gw, 61.46.Df

\section{INTRODUCTION}

The magnetic anisotropy of assembled nanoparticles (NPs) systems constitutes a key parameter in the understanding of their magnetic behavior, as well as in their possible technological applications [1]. Current research is performed towards the control and tailoring of this parameter using diverse strategies. Over the last years, we have performed an intense research in this direction by exploring enhanced magnetic anisotropy in different cobalt granular systems [2-7]. These granular films consist of self-organized lattices of nanometer-sized Co magnetic clusters (1-4 $\mathrm{nm}$ in diameter) prepared by sequential deposition of $\mathrm{Al}_{2} \mathrm{O}_{3}$ and Co layers on a Si substrate. Our investigation allowed quantitative determination of their magnetic anisotropy [3,4] and dipolar interactions [2], as well as the control of their surface anisotropy by capping the cobalt NPs with noble metals like $M=\mathrm{Cu}, \mathrm{Ag}$, and $\mathrm{Au}$ [5]. A systematic increase in the surface anisotropy of these Co- $M$ NPs with capping in the trend $\mathrm{Cu}, \mathrm{Ag}$, and $\mathrm{Au}$ was then observed, as resulting from the orbital moment anisotropy, caused by hybridization of Co atoms at the particle surface with the capping metal [6].

However, the induction of superficial changes is not the only way of tailoring the anisotropy of these Co granular systems. Other routes recently used for this purpose consist in producing alloyed particles, so that the intrinsic source of anisotropy is modified [7-12]. In this respect, we have recently explored the introduction of high spin-orbit nonmagnetic elements and

\footnotetext{
*aifigueg@gmail.com
}

induce their alloying with Co. In particular, we have studied granular films of Co capped with W [7] and Pt [12].

On the one hand, W has a known affinity to alloy with Co on sputtering grown films at room temperature [13], and these alloys tend to become amorphous [14-17]. Therefore the Co-W NPs is a system of amorphous alloyed particles, which constitute a different type with variable anisotropy, induced by alloying, irrespective of surface effects [7]. Their magnetic anisotropy properties were determined as result of the shortrange order revealed in the $\mathrm{Co}-\mathrm{W}$ alloy.

On the other hand, Pt has also a large affinity to alloy with Co. In fact, the production of Co-Pt alloys is one of the preferred routes currently used in nanoparticles and thin films to increase their magnetic anisotropy [8-10]. For example, CoPt NPs embedded in different matrices show enhanced magnetic anisotropy with respect to the Co particles $[9,10,18,19]$. This results from the crystallization of Co-Pt alloys in the chemically ordered $L 1_{0}$ phase, which, in bulk form, has a very high magnetocrystalline anisotropy [20]. In addition, many Co-Pt multilayer systems [21-24], $\mathrm{Co}_{x} \mathrm{Pt}_{1-x}$ alloy films [11,25-27], and nanopatterned Co-Pt systems [28] show perpendicular magnetic anisotropy (PMA): a property that favors the easy axis of magnetization along the film normal direction. This PMA property is interesting for applications in spintronic nanodevices, for example, in ultrahigh-density magnetic storage, fast memory applications, and nanosensors $[29,30]$. The origin of PMA is also correlated with the Co orbital anisotropy, which by hybridization with $\mathrm{Pt}$, and due to the high spin-orbit coupling of the latter, gives rise to an enhanced out of plane anisotropy [10,20,31]. Most of the Co-Pt systems reported in the literature are either produced at high temperatures or annealed in order to favor the crystallization 
of the Co-Pt alloy in this anisotropic $L 1_{0}$ phase. However, the origin of PMA in Co-Pt alloy systems deposited at room temperature (RT) remains controversial [11].

In order to deepen in the understanding of the PMA properties in $\mathrm{Co}-\mathrm{Pt}$ particulate systems prepared by $\mathrm{RT}$ deposition, we have performed a systematic study of the morphological, structural and magnetic properties of $\mathrm{Co}-\mathrm{Pt}$ granular multilayers prepared by RT sequential sputtering deposition of $\mathrm{Al}_{2} \mathrm{O}_{3}, \mathrm{Co}$, and $\mathrm{Pt}$ on a $\mathrm{Si}$ substrate. Previous studies of Co-Pt alloy nanostructured systems have demonstrated the importance of a complete investigation of both the local atomic environment and their long range order to clarify the correlation between crystallographic structure and magnetic anisotropy [11]. Thus we examine the role of Co-Pt alloying in the origin of the magnetic anisotropy in these Co-Pt granular films. Their morphology and local structure are studied by high-resolution transmission electron microscopy (HRTEM) and extended x-ray absorption fine structure (EXAFS). The magnetic characterization includes macroscopic measurements performed by SQUID and anomalous Hall effect (AHE) magnetometries, and microscopic measurements by x-ray magnetic circular dichroism (XMCD). In addition, we include angle dependent XMCD measurements, which constitute an effective method to explore the anisotropy of the orbital moment in the magnetism of Co-Pt systems with PMA [27,32].

\section{SAMPLES STUDIED}

The samples studied were prepared at the Unité Mixte de Physique CNRS/Thales by sequential sputtering deposition of $\mathrm{Al}_{2} \mathrm{O}_{3}, \mathrm{Co}$, and $\mathrm{Pt}$ on a $\mathrm{Si}$ substrate, following the same procedure as it is described in previous works on metal capped Co nanoparticles $[6,7]$. The alumina, cobalt, and platinum are deposited using Ar plasma, the metals in dc mode and the insulator at a RF power of $2.2 \mathrm{~W} / \mathrm{cm}^{2}$. The substrate temperature is kept constant at $293 \mathrm{~K}$ and the Ar pressure is $2 \times 10^{-3}$ Torr. The formation of Co aggregates on the amorphous alumina is the result of three-dimensional growth because of the different surface energies between alumina and Co [33]. Aggregation occurs below a certain threshold of the nominal thickness of $\mathrm{Co}, t_{\mathrm{Co}}$, that the layer would have if it were continuous. Previous studies show that not all the Co deposited on the alumina layer aggregates as forming the particles, but there exists a fraction of Co atoms or small clusters dispersed on the matrix, defined as $x_{\text {para }}$ [34]. The values of $x_{\text {para }}$ depend on $t_{\text {Co }}$, varying between 0.5 and 0.13 for $t_{\text {Co }}=0.4$ and $1.0 \mathrm{~nm}$, respectively [34]. The Co clusters are subsequently capped with a Pt layer, for which the nominal thickness is denoted by $t_{\mathrm{Pt}}$. A new alumina layer of about $3 \mathrm{~nm}$ is deposited on top of this Co-Pt system. This sequential deposition process is repeated $N$ times in order to get a granular multilayer system, following the formula $\mathrm{Al}_{2} \mathrm{O}_{3} /\left(\mathrm{Al}_{2} \mathrm{O}_{3} / \mathrm{Co} / \mathrm{Pt}\right)_{N} / \mathrm{Al}_{2} \mathrm{O}_{3}$.

In this manuscript, we describe the characterization of granular Co-Pt film samples with a fixed $t_{\mathrm{Co}}=0.7 \mathrm{~nm}$, varying $t_{\mathrm{Pt}}=0.6,1.5,4.5$, and $6.0 \mathrm{~nm}$, and $N=25$ in all cases. For the sake of comparison, a reference sample of "bare" (noncapped) Co nanoparticles embedded in amorphous alumina layers of $t_{\mathrm{Co}}=0.7 \mathrm{~nm}$ and $t_{\mathrm{Al}_{2} \mathrm{O}_{3}}=3 \mathrm{~nm}$, with average diameter $\langle D\rangle \approx 3 \mathrm{~nm}$ in a sequence of $N=20$ repetitions, has been included in this study [34].

\section{EXPERIMENTAL DETAILS}

The morphology of the granular Co-Pt multilayer samples has been studied by HRTEM and high-angle annular dark field (HAADF) scanning transmission electron microscopy (STEM) measurements. HRTEM images were taken in both cross section and plan view configurations, in a JEOL JEM 3000 F Field Emission Microscope fitted with an Oxford LINK Electron Dispersive Spectrometry (EDS) analyzer.

X-ray absorption (XAS) and EXAFS spectra for the granular Co-Pt films were measured at the Co $K(7709 \mathrm{eV})$ and $\mathrm{Pt}$ $L_{3}(11564 \mathrm{eV})$ edges using a double-crystal Si(111) monocromator in the BM29 beamline at the European Synchrotron Radiation Facility (ESRF). Measurements were performed at $300 \mathrm{~K}$ in fluorescence detection mode with a 13-element Ge solid state detector with digital signal processing for fluorescence XAS, high-energy resolution and high count rate. Reference samples were also measured at different edges: the "bare" Co NPs with an average diameter of $3 \mathrm{~nm}$, and the Co metallic foil measured at the Co $K$ edge; and Pt metallic foil measured at the Pt $L_{3}$ edge. For each edge measured on each sample, four spectra with a 4 s/point count were taken in order to have enough statistics in data treatment.

XANES and XMCD measurements at the Co $K(7709 \mathrm{eV})$, Pt $L_{3}(11564 \mathrm{eV})$, and $\mathrm{Pt} L_{2}(13273 \mathrm{eV})$ edges on the Co-Pt granular film with $t_{\mathrm{Co}}=0.7 \mathrm{~nm}$ and $t_{\mathrm{Pt}}=1.5 \mathrm{~nm}$ were performed at the ESRF ID12 beamline. The APPLEII undulator and a double Si (111) crystal monocromator were used to collect the spectra at the respective energies. XANES spectra were recorded by a fluorescence detector in backscattering geometry. Polarization of the circular light was over $90 \%$ in these cases. Angle dependent XANES and XMCD experiments at the Co $L_{3}(778.1 \mathrm{eV})$ and Co $L_{2}(793.2 \mathrm{eV})$ edges on the same Co-Pt granular sample were performed at the ID08 beamline of the ESRF, with incident angles $\theta=0^{\circ}$ and $60^{\circ}$ with respect to the sample surface normal. An APPLE-II undulator and a spherical grating monochromator were used in this case. The degree of polarization at these Co $L_{2,3}$ edges was $\sim 100 \%$ and the total electron yield (TEY) detection method was employed. The XMCD signal at each edge was obtained by applying a magnetic field of $10 \mathrm{kOe}$ along the x-ray beam direction, at a temperature of $10 \mathrm{~K}$. This field and temperature were chosen according to the magnetic properties of the Co-Pt granular multilayer, so that the system was reaching its magnetic saturation under these conditions. XMCD was obtained by differences of XANES spectra measured with opposite helicities of the light at a fixed magnetic field value, orienting the field in two inverse directions.

Magnetization measurements were performed with a SQUID magnetometer equipped with the high-resolution (RSO) option. Both magnetization as a function of the applied field, $M(H)$, and as a function of temperature, $M(T)$, were measured in two configurations: (1) with the field applied parallel, and (2) perpendicular to the substrate plane. The $M(T)$ curves were measured after zero-field cooling (ZFC) and field cooling (FC) the sample. Temperature was varied between 
5 and $400 \mathrm{~K}$, and a $0.2 \mathrm{kOe}$ field was applied. Hysteresis loops were measured at several temperatures from 5 to $350 \mathrm{~K}$, under applied fields up to $50 \mathrm{kOe}$.

Anomalous Hall effect (AHE) measurements were performed on two of the Co-Pt samples, those with $t_{\mathrm{Co}}=0.7 \mathrm{~nm}$, and $t_{\mathrm{Pt}}=1.5$ and $6.0 \mathrm{~nm}$. These measurements were carried out in a home made electrical transport setup and in a Quantum Design Physical Property Measurement System (PPMS). A six-probe method was employed with the contacts bonded by wire welding and microcontacting at the edges of the rectangular samples, for the measurements in the home made electrical transport setup and the PPMS, respectively. The magnetic field was varied within $H= \pm 20 \mathrm{kOe}$, and applied in the direction perpendicular the substrate. $V_{H}$ versus $H$ loops were collected at several temperatures from 2 to $300 \mathrm{~K}$.

\section{MORPHOLOGICAL AND STRUCTURAL PROPERTIES}

\section{A. Microscopy measurements}

Low-magnification studies in cross-section configuration for all four samples confirmed the presence of 25 layers in agreement to the nominal layer preparation. HRTEM images, shown in Fig. 1, and EDS analysis indicate that light gray layers correspond to the amorphous alumina and darker layers to the $\mathrm{Co}$ and $\mathrm{Pt}$ metals. Each Co-Pt metallic layer is 2.3-, 2.5-, 5.5-, and 6.7-nm thick, for the samples with $t_{\mathrm{Pt}}=0.6,1.5,4.5$, and $6.0 \mathrm{~nm}$, respectively. These thicknesses evidence particulate morphology for the samples with the lowest amount of $\mathrm{Pt}\left(t_{\mathrm{Pt}}=\right.$ 0.6 and $1.5 \mathrm{~nm}$ ) given that they are greater than the sum of the nominal thickness of $t_{\mathrm{Co}}$ and $t_{\mathrm{Pt}}$ in the sample: $0.7+0.6 \leqslant 2.3$ and $0.7+1.5 \leqslant 2.5 \mathrm{~nm}$, respectively. This is similar to what is observed for bare Co NPs in Ref. [35]. In Figs. 1(a) and 1(b), there are indeed some signs of particulated morphology, as in

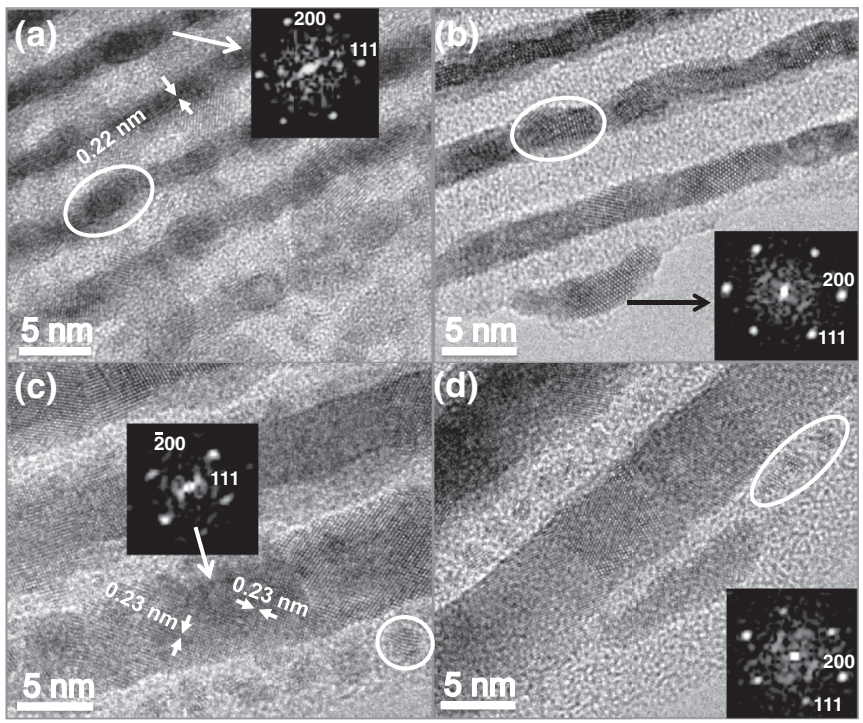

FIG. 1. HRTEM images in cross section configuration for samples with $t_{\mathrm{Co}}=0.7 \mathrm{~nm}$ and (a) $t_{\mathrm{Pt}}=0.6$, (b) 1.5 , (c) 4.5 , and (d) $6.0 \mathrm{~nm}$. Fast Fourier transform (FFT) at selected zones of the images are shown, with the spots indexed according to the interplanar distances of a fcc cell in all cases. A Co-Pt particle is highlighted in (a) and (b). Pt NPs are marked in (c) and (d).

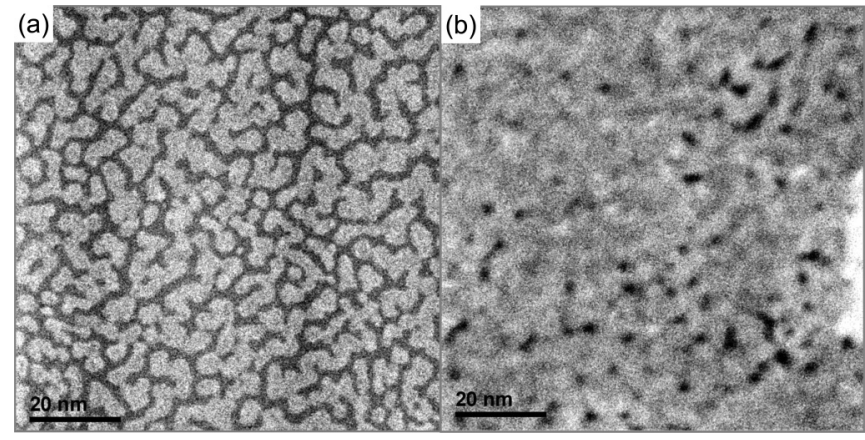

FIG. 2. STEM images in plan view configuration for the samples with $t_{\mathrm{Co}}=0.7 \mathrm{~nm}$, and (a) $t_{\mathrm{Pt}}=0.6$ and (b) $1.5 \mathrm{~nm}$.

the region surrounded by a white ellipse. Some darker shadows inside the layers indicate the presence of $\mathrm{Co}$, located at the center of the layer, suggesting that the capping $\mathrm{Pt}$ tends to surround the Co particle. For the other two samples $\left(t_{\mathrm{Pt}}=\right.$ 4.5 and $6.0 \mathrm{~nm}$ ), however, the Co-Pt nanoparticles are not easy to identify from the images obtained in cross section configuration.

Crystallinity in the Co-Pt granular films is observed in all four samples studied by HRTEM. Fast Fourier transform (FFT) performed on selected areas of the images shows spots that are well indexed with the interplanar distances of a fcc cell in all cases (see insets in Fig. 1). In Figs. 1(a) and 1(c), the periodicity found in the selected zone of the image is $0.22-0.23$ $\mathrm{nm}$, which is characteristic of the (lll 111$)$ planes of a fcc Pt structure. In Figs. 1(c) and 1(d), some small crystalline clusters in the alumina region of the samples are observed (see circled particles in the figures), which have been identified as Pt clusters with fcc structure, ranging between 2 and $3 \mathrm{~nm}$ in diameter.

Improved contrast to better identify the Co-Pt particles is obtained by plan view STEM images, such as the one shown for the sample with $t_{\mathrm{Co}}=0.7 \mathrm{~nm}$ and $t_{\mathrm{Pt}}=0.6 \mathrm{~nm}$ in Fig. 2(a). In this image, brighter parts correspond to Co-Pt, and darker parts to $\mathrm{Al}_{2} \mathrm{O}_{3}$. The image obtained is comparable to those of similar systems of metallic nanoparticles embedded in insulator matrices [36], and the shape of the particles is similar to that of Co-nanoparticles embedded in alumina [35]. The average diameter of the Co-Pt grains from the image in Fig. 2(a) was 4.1(2) nm. STEM images for the other three samples (those with $t_{\mathrm{Pt}}=1.5,4.5$, and $6.0 \mathrm{~nm}$ ) did not allow identification of the Co-Pt particles since they revealed an almost uniform Pt capping, as shown in Fig. 2(b) for the sample with $t_{\mathrm{Pt}}=1.5 \mathrm{~nm}$.

\section{B. XANES and EXAFS measurements}

The $\mathrm{x}$-ray absorption spectra were processed and analysed using different tools of the IFFEFIT [37] XAS package. It included preliminary reduction of the EXAFS raw data involving background removal of the $\mathrm{x}$-ray absorption data $\mu(\mathrm{E})$, conversion of $\mu(\mathrm{E})$ to $\chi(\mathrm{k})$, normalization and weighting scheme, which were all performed with AUTOBK and ATHENA. EXAFS data analysis and fitting on all reference and samples at their respective edges were performed in ARTEMIS, making use of models based on crystallographic information found in 


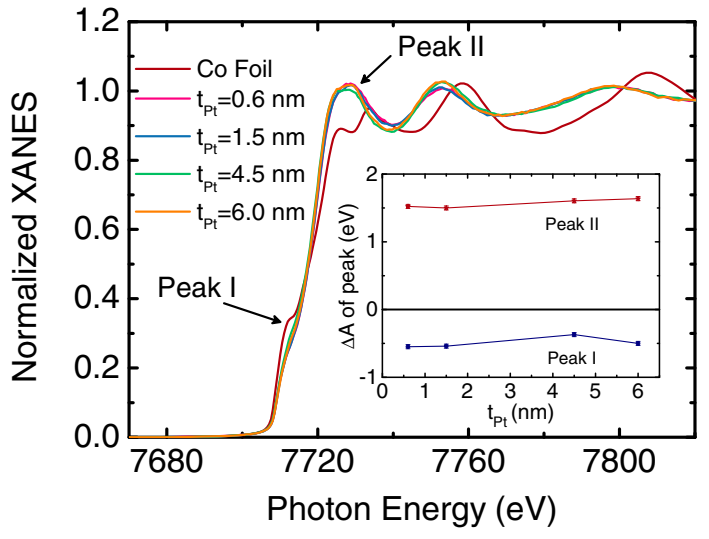

FIG. 3. (Color online) XANES at the Co $K$ edge for the Co-Pt granular films and comparison with that of a Co foil for reference. (Inset) Variation of the areas of peaks I and II in the figure as a function of $t_{\mathrm{Pt}}$.

the ICSD [38] database; atomic clusters used to generate the scattering paths for fitting were generated with ATOMS [39].

\section{Co K edge}

Normalized x-ray absorption near edge structure (XANES) spectra at the Co $K$ edge for Co-Pt granular films with $t_{\mathrm{Co}}=$ $0.7 \mathrm{~nm}$ and $t_{\mathrm{Pt}}=0.6,1.5,4.5$, and $6.0 \mathrm{~nm}$ are shown in Fig. 3, along with the Co foil XANES, plotted for reference. Changes in the intensity and shape of the pre-edge peak and the white line (denoted as peak I and peak II, respectively, in Fig. 3) are appreciable and reflect changes in the electronic structure of the Co (electron configuration $[\mathrm{Ar}] 3 d^{7} 4 s^{2}$ ) and $\mathrm{Pt}$ (electron configuration $\left.[\mathrm{Xe}] 4 f^{14} 5 d^{9} 6 s^{1}\right)$ atoms. We have calculated the difference of the peaks areas, with respect to that of the Co foil reference, as $\Delta A=\int_{E_{i}}^{E_{f}}\left(\mu_{\text {sample }}-\mu_{\text {Co-foil }}\right) d E$. The inset of Fig. 3 represents the $\Delta A$ as a function of the Pt content in the samples. The integration limits were 7706.1-7717.6 eV for peak I, and 7717.6-7735.1 eV for peak II.

Let us recall that the Co $K$-edge white line reflects the $4 p$ empty states of Co near the Fermi energy, while the pre-edge feature (labelled peak I) arises from transitions to more localized unoccupied $3 d$ states, influenced by Co $4 p-3 d$ hybridization. The reduction of the intensity of peak I in the Co-Pt samples with respect to that of the Co foil reveals a delocalization of the Co $3 d$ empty states, and is a proof of Co $4 p-3 d-$ Pt $5 d$ hybridization.

The intensity of peak II depends on both the number of available $4 p$ states, and the structure of the system. It is evident that the intensity of this peak II is higher and its shape different from that of the Co foil. Indeed, it looks closer to the shape and intensity of that recorded for bulk Co-Pt alloys in Ref. [40], which is an indication of the presence of such kind of alloys in our Co-Pt granular films. In any case, it is clear that the $4 p$ empty states in the Co-Pt granular samples increase, compared to those of the bulk Co.

Both peak I and peak II are practically constant along the Co-Pt sample series (see inset of Fig. 3), reflecting that Co $3 d-\mathrm{Pt} 5 d$ hybridization is not affected by such variations in the amount of Pt for a fixed Co content. This fact strongly suggests that the alloy composition in the Co-Pt granular samples is
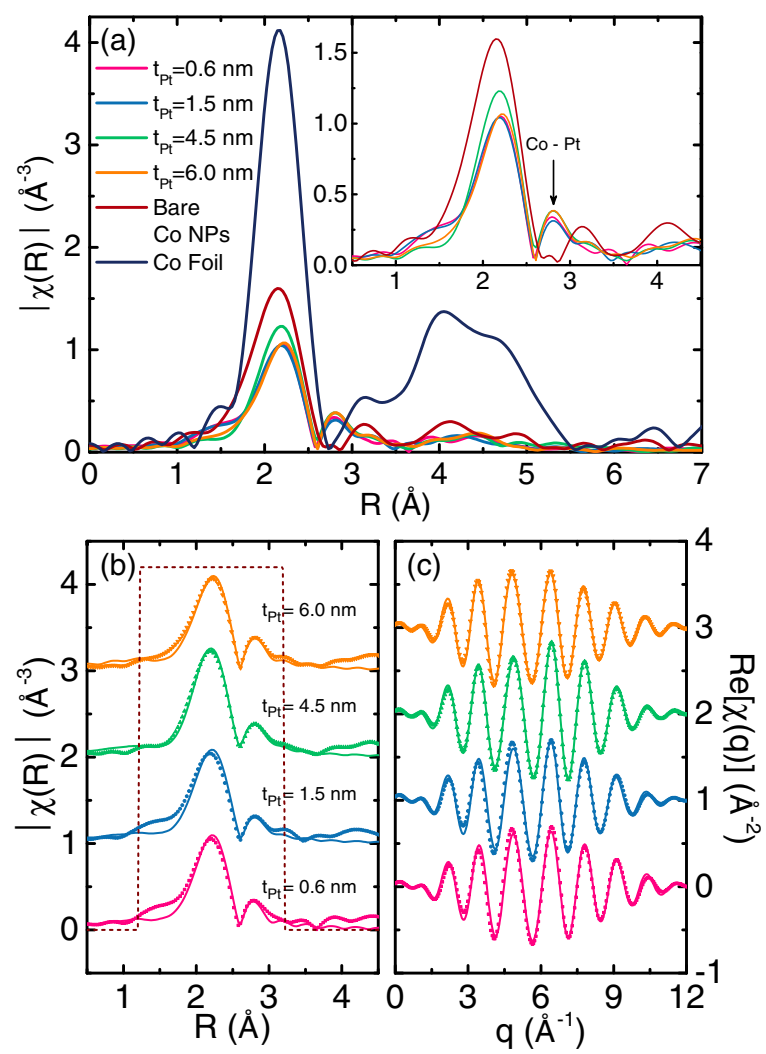

FIG. 4. (Color online) (a) Fourier transform of EXAFS signal at the Co $K$ edge on the Co-Pt samples. The measurements on the Co foil and bare Co nanoparticles with $t_{\mathrm{Co}}=0.7 \mathrm{~nm}$ in are shown for comparison. (Inset) Zoom of the first coordination shell in the particulate systems. (b) Best fits to the first coordination shell in (a) for the Co-Pt nanoparticles in R space. (c) Contribution of the first coordination shell to the EXAFS signal for the Co-Pt nanoparticles along with its best fit. In (b) and (c), symbols represent experimental data and solid lines their best fits; curves have been vertically shifted for clarity.

similar for these four samples studied, a result that will be confirmed by EXAFS (later in this section).

The structural analysis of the Co-Pt granular films that can be extracted from the $\chi(\mathrm{k})$ EXAFS signal is better studied by its Fourier transform (FT), shown in Fig. 4(a), performed on a $k$ range from 2.4 to $10.6 \AA^{-1}$ using a $k^{2}$ weight and a Kaiser-Bessel window function. This Co $K$-edge $R$-space data show peaks that correspond to different atomic shells surrounding the Co atoms in the samples. We have plotted the FT EXAFS of Co-Pt granular samples along with the Co foil and bare Co NPs for comparison. The Co environment of the latter two reference samples is very similar, which is revealed from the same position and shape of the peaks, not only for the first coordination shell, but also for outer shells. However, the peaks for outer coordination shells are different in the Co-Pt samples [see Fig. 4(a) and inset]. Indeed, the appearance of a peak characteristic of a Co-Pt distance is identified in all four samples, and is marked in the inset of Fig. 4(a). The intensities of all peaks of the FT EXAFS are larger in the Co foil than in the particulate systems, as it is expected from the reduction in the mean Co coordination due to the size reduction. 
TABLE I. Structural parameters obtained from the Co $K$-edge EXAFS fits for the Co-Pt NPs shown in Fig. 4. Coordination numbers $N$, interatomic distance $R$, and Debye-Waller factor $\sigma^{2}$ for each path used in the fitting model.

\begin{tabular}{lccccccc}
\hline \hline & \multicolumn{3}{c}{ Co-Co } & & \multicolumn{3}{c}{ Co-Pt } \\
\cline { 2 - 3 } $\begin{array}{l}t_{\mathrm{Pt}} \\
(\mathrm{nm})\end{array}$ & $N_{\mathrm{Co}-\mathrm{Co}}$ & $\begin{array}{c}R_{\mathrm{Co}-\mathrm{Co}} \\
(\AA)\end{array}$ & $\begin{array}{c}\sigma_{\mathrm{Co}-\mathrm{Co}}^{2} \\
\left(\AA^{2}\right)\end{array}$ & & $N_{\mathrm{Co}-\mathrm{Pt}}$ & $\begin{array}{c}R_{\mathrm{Co}-\mathrm{Pt}} \\
(\AA)\end{array}$ & $\begin{array}{c}\sigma_{\mathrm{Co}-\mathrm{Pt}}^{2} \\
\left(\AA^{2}\right)\end{array}$ \\
\hline 0.6 & $5.4(5)$ & $2.57(2)$ & $0.017(2)$ & $3.7(5)$ & $2.61(2)$ & $0.009(2)$ \\
1.5 & $5.3(5)$ & $2.57(2)$ & $0.016(2)$ & & $4.2(5)$ & $2.63(2)$ & $0.010(2)$ \\
4.5 & $4.7(5)$ & $2.55(2)$ & $0.013(2)$ & & $5.5(5)$ & $2.63(2)$ & $0.012(2)$ \\
6.0 & $6.3(5)$ & $2.59(2)$ & $0.021(2)$ & $4.9(5)$ & $2.64(2)$ & $0.010(2)$ \\
\hline \hline
\end{tabular}

From a qualitative analysis of the peaks shape and position of the FT EXAFS at the Co $K$ edge for the four Co-Pt NPs, it is noticeable that the Co neighborhood is very similar in all four samples. This was confirmed by the fitting of EXAFS signal, performed on the $R$-space in a range from 1.2 to $3.2 \AA$ using a Kaiser-Bessel window function, so that it covered the Co first coordination shell [see Figs. 4(b) and 4(c)]. Different models were tested in order to fit the Co $K$-edge $R$-space data. The best fits were achieved with two near neighbor distances: a Co-Co path around $2.56 \AA$ and a Co-Pt path around $2.63 \AA$ (according to the ICSD [38]).

The structural parameters for fitting the $R$-space EXAFS at the Co $K$ edge of each Co-Pt NPs sample were the average coordination $(N)$, interatomic distance $(R)$, and the DebyeWaller factor $\left(\sigma^{2}\right)$ for each path. The amplitude reduction factor $\mathrm{S}_{0}^{2}$ and the shift in the threshold energy $\Delta E_{0}$ were fixed to those obtained for the Co foil fit $\left(\mathrm{S}_{0}^{2}=0.72\right.$ and $\left.\Delta E_{0}=7 \mathrm{eV}\right)$. A total of six fitting parameters were used for an iterative procedure, which consistently included the fitting of both Co $K$ and Pt $L_{3}$ edges (see Sec. IVB2). Results for the Co $K$-edge EXAFS fits for the Co-Pt NPs are shown in Figs. 4(b) and 4(c), and the values obtained for each sample are listed in Table I.

The Co-Co and Co-Pt distances obtained in the samples are very similar for all of them. These distances have been identified to belong to a $\mathrm{Co}_{x} \mathrm{Pt}_{1-x}$ alloy with composition close to that with $x=0.5$, by comparison to the Co $K$-edge EXAFS results in Co-Pt films with that composition [11] and annealed Co-Pt alloy nanoparticles with $L 1_{0}$ structure with sizes comparable to ours [41]. Such distances, as well as the coordination and Debye-Waller factors for each path, are almost constant along the series, which indicates that the Co-Pt alloy in the samples has the same or very close composition. The Debye-Waller factors, however, are rather high, which may be a signature of significant dispersion of the Co-Co and Co-Pt distances. Nevertheless, attempts to include additional distances in the model for the EXAFS fit yielded to unclear results.

The EXAFS results of distances and coordination numbers in our Co-Pt granular samples differ from those of chemically synthesized Co-core Pt-shell NPs of comparable sizes [42], e.g., the Co-Co distance being longer and the coordination lower in our case. This result demonstrates that the structure of our particles is different from that of a simple core-shell particle with a Co core and a Pt shell. However, there might be a presence of $\mathrm{Co}-\mathrm{Co}$ bonds belonging to metallic $\mathrm{Co}$ (as in a $\mathrm{Co}$ core) in our Co-Pt granular samples, given the high dispersion of distances found in our results, as described above.

The crystal structure of the Co-Pt alloy in these granular films can also be analyzed from the values of the $N_{\mathrm{Co}-\mathrm{Pt}} / N_{\mathrm{Co}-\mathrm{Co}}$ ratio, following the procedure described in Ref. [41]. A value of $N_{\text {Co-Pt }} / N_{\text {Co-Co }}=1$ is indicative of a chemically disordered $A 1$ crystal structure, while $N_{\mathrm{Co}-\mathrm{Pt}} / N_{\mathrm{Co}-\mathrm{Co}}=2$ is characteristic of a $L 1_{0}$ ordered structure. In our Co-Pt granular films, if we consider $40 \%$ of Co-Co bounds involved in the alloyed interface, we found $N_{\mathrm{Co}-\mathrm{Pt}} / N_{\mathrm{Co}-\mathrm{Co}}=2$ as expected for a crystal structure close to that of the ordered $L 1_{0} \mathrm{Co}-\mathrm{Pt}$ alloy. In addition, the tetragonalization ratio $c / a=\sqrt{2\left(R_{\mathrm{Co}-\mathrm{Pt}} / R_{\mathrm{Co}-\mathrm{Co}}\right)^{2}-1}$ is larger than 1 for all samples, with values comparable to those found for annealed Co-Pt alloy nanoparticles with $L 1_{0}$ structure of comparable size in Ref. [41].

\section{Pt $L_{3}$ edge}

Normalized XANES spectra at the Pt $L_{3}$ edge for the Co-Pt granular films samples are shown in Fig. 5, along with that of a Pt foil at the same edge for comparison. Since Pt is a $5 d$ metal, the $L_{3}$ absorption edge appears for transitions from $2 p$ to both $5 d_{3 / 2}$ and $5 d_{5 / 2}$ bands. In general, the white line feature of the Pt $L_{3}$ in the Co-Pt granular samples in Fig. 5 is reduced with respect to that of the $\mathrm{Pt}$ foil as the amount of $\mathrm{Pt}$ in the sample decreases. The same trend is also observed for Co-Pt alloys in Ref. [40], as the Co concentration in the alloy increases. A decrease in the $\mathrm{Pt} L_{3}$ edge intensity from one sample to the other shows a decrease in the number of Pt $5 d$ available empty states. Such a decrease may be understood as an electron transfer from Co towards $\mathrm{Pt}$, due to hybridization between the Pt $5 d$ and Co $4 p-3 d$ electronic states.

It is evident that those samples with high amount of $\mathrm{Pt}$, namely $t_{\mathrm{Pt}}=4.5$ and $6.0 \mathrm{~nm}$, on one hand, have a spectrum very close in shape and intensity to that of the Pt foil, showing a higher amount of metallic Pt in these two samples. On the other hand, the XANES of the samples with low amount of $\mathrm{Pt}$ $\left(t_{\mathrm{Pt}}=0.6\right.$ and $\left.1.5 \mathrm{~nm}\right)$ differ from that of the Pt foil, showing a broader and less intense white line, which is an indication of

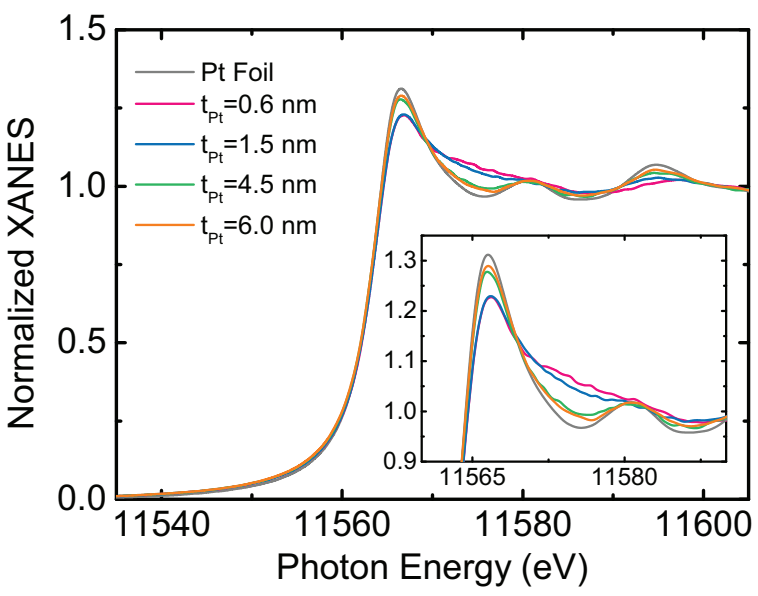

FIG. 5. (Color online) XANES spectra at the Pt $L_{3}$ edge for the Co-Pt granular multilayer samples, along with the absorption of the Pt foil plotted for reference. (Inset) Details of the variation of the white line intensity. 

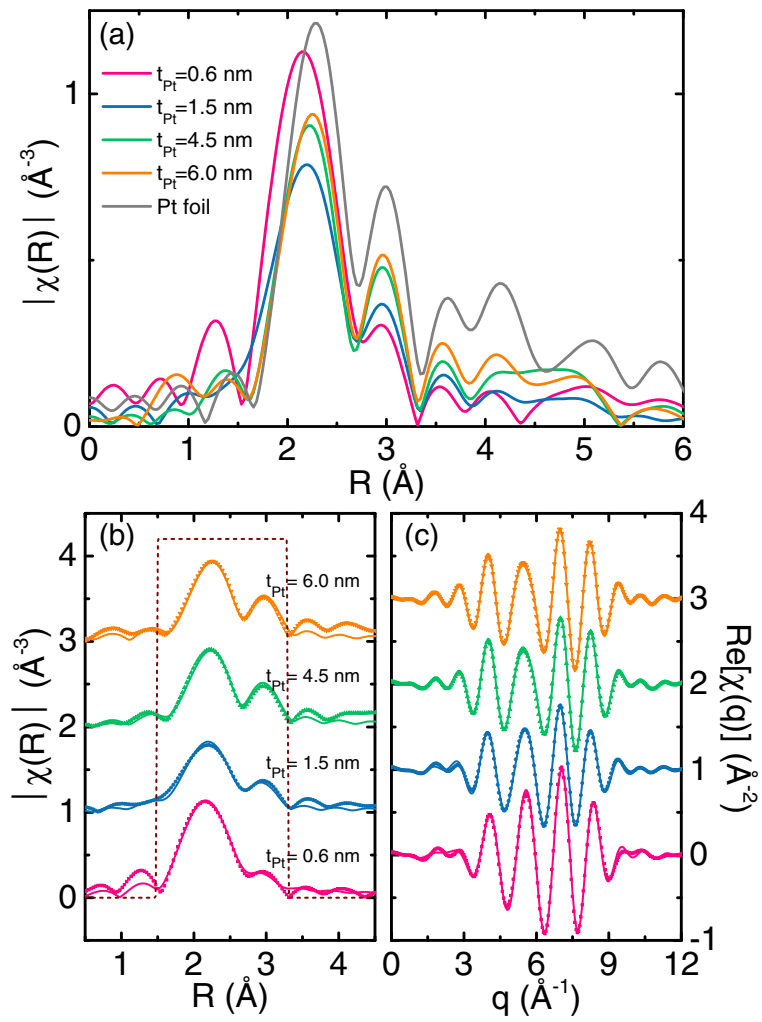

FIG. 6. (Color online) (a) Fourier transform of EXAFS signal at the $\mathrm{Pt} L_{3}$ edge on the Co-Pt granular films studied and comparison with that of the Pt foil. (b) Best fits to the first coordination shell in (a) for the Co-Pt nanoparticles in R space. (c) Contribution of the first coordination shell to the EXAFS signal for the Co-Pt nanoparticles along with their best fits. In (b) and (c) symbols represent experimental data and solid lines their best fits; curves have been vertically shifted for clarity.

different electronic structure and Pt environment. Later in this section, we will demonstrate by EXAFS analysis performed in these samples how the Pt environment differs, being very similar to the metallic Pt in the other two mentioned samples $\left(t_{\mathrm{Pt}}=4.5\right.$ and $\left.6.0 \mathrm{~nm}\right)$.

The Fourier transformed EXAFS signals at the Pt $L_{3}$ of the Co-Pt granular films, along with that of the $\mathrm{Pt}$ foil used for reference, are shown in Fig. 6(a). The $k$ range for the FT goes from 2.7 to $8.5 \AA^{-1}$ using a $k^{2}$ weight and a Kaiser-Bessel window function. A qualitative inspection of the peaks shows, again, that the Pt environment in the samples with $t_{\mathrm{Pt}}=4.5$ and $6.0 \mathrm{~nm}$ is very similar to that of the metallic $\mathrm{Pt}$.

The Pt $L_{3}$ EXAFS fitting was performed on the $R$ space in a $R$ range from 1.5 to $3.3 \AA$, using a Kaiser-Bessel window function. $S_{0}^{2}$ and $\Delta E_{0}$ were set to those values obtained for the Pt foil EXAFS fit $\left(S_{0}^{2}=0.72\right.$ and $\left.\Delta E_{0}=8 \mathrm{eV}\right)$. The Pt $L_{3}$ EXAFS fitting model for the Co-Pt granular samples included a combination of a Co-Pt distance from a Co-Pt alloy, and a Pt-Pt distance from the metallic Pt (according to the ICSD [38]). The fitting parameters were six, in total, for each sample, in an iterative procedure including the fitting of both Co $K$ and Pt $L_{3}$ edges, as it was previously described.

The EXAFS fitting results for the Co-Pt granular samples are plotted in Fig. 6(b) and the values listed in Table II. The
TABLE II. Structural parameters obtained from the $\mathrm{Pt} L_{3}$-edge EXAFS fits for the Co-Pt granular samples shown in Fig. 6(b). Coordination numbers $N$, interatomic distance $R$, and Debye-Waller factor $\sigma^{2}$ for both paths used in the fitting model.

\begin{tabular}{lccccccc}
\hline \hline & \multicolumn{3}{c}{$\mathrm{Pt}-\mathrm{Co}$} \\
\cline { 3 - 4 } \cline { 6 - 8 } $\begin{array}{l}t_{\mathrm{Pt}} \\
(\mathrm{nm})\end{array}$ & $N_{\mathrm{Pt}-\mathrm{Co}}$ & $\begin{array}{c}R_{\mathrm{Pt}-\mathrm{Co}} \\
(\AA)\end{array}$ & $\begin{array}{c}\sigma_{\mathrm{Pt}-\mathrm{Co}}^{2} \\
\left(\AA^{2}\right)\end{array}$ & & $N_{\mathrm{Pt}-\mathrm{Pt}}$ & $\begin{array}{c}R_{\mathrm{Pt}-\mathrm{Pt}} \\
(\AA)\end{array}$ & $\begin{array}{c}\sigma_{\mathrm{Pt}-\mathrm{Pt}}^{2} \\
\left(\AA^{2}\right)\end{array}$ \\
\hline 0.6 & $4.6(9)$ & $2.62(3)$ & $0.008(3)$ & & $4.7(9)$ & $2.65(3)$ & $0.004(4)$ \\
1.5 & $2.4(5)$ & $2.62(2)$ & $0.012(2)$ & & $9.3(5)$ & $2.73(2)$ & $0.008(4)$ \\
4.5 & - & - & - & & $11.8(5)$ & $2.73(1)$ & $0.006(1)$ \\
6.0 & - & - & - & $11.8(5)$ & $2.74(1)$ & $0.006(1)$ \\
\hline \hline
\end{tabular}

Pt-Co distance of $2.62 \AA$ found for the samples with $t_{\mathrm{Pt}}=0.6$ and $1.5 \mathrm{~nm}$ are well in agreement with the results found for the same samples at the Co $K$-edge EXAFS analysis (see Table I), which confirms the presence of a Co-Pt alloy in the samples.

The sample with the least amount of $\mathrm{Pt}, t_{\mathrm{Pt}}=0.6 \mathrm{~nm}$, has a Pt-Pt environment quite different from the other three samples. The Pt-Pt distance is in this case a bit shorter $(2.65 \AA)$ than those found for the others $(\sim 2.73 \AA)$, which might indicate that Pt is not in its metallic form. Such a short Pt-Pt distance has been detected in electrochemically deposited fcc $\mathrm{Co}-\mathrm{Pt}$ alloys [43]. Then, it is very likely that most of the deposited Pt in this sample may be alloyed with the Co atoms. which also agrees well with the crystal structure observed for the particles by HRTEM image in Fig. 1(a).

The EXAFS signals of the samples with the highest amount of Pt, namely, $t_{\mathrm{Pt}}=4.5$ and $6.0 \mathrm{~nm}$, were fitted only with a Pt-Pt model since introducing a Co-Pt distance had no effect in their fit. From the fitting results in these two samples, we found the same structure around the Pt atoms, as it was observed from the $\mathrm{Pt} L_{3}$ XANES, being very close to that of the Pt foil. In fact, the Pt-Pt coordination being close to 12 , and interatomic distances of 2.73-2.74 $\AA$ are close to those obtained for the Pt foil used as a reference with fcc structure.

Similar Co-Co, Co-Pt, and Pt-Pt distances to those found for our Co-Pt granular multilayers have been reported for Co-clusters of similar sizes $(\sim 3 \mathrm{~nm})$, prepared by low energy cluster beam deposition (LECBD), embedded in a Pt matrix, where diffusion of the atoms at the surface cluster takes place, resulting in a Pt-Co alloyed interface of one or two monolayers [44]. Analogous processes may be present in our Co-Pt granular samples. However, the coordination numbers we have obtained for the Co-Pt and Pt-Pt environments are much higher than those listed in Ref. [44], which may indicate that the Co-Pt alloy interface in our $\mathrm{Co}-\mathrm{Pt}$ granular films extends to more layers at the Co-core surface.

Summarizing, the morphological and structural characterization of the Co-Pt granular multilayers reveal the presence of a Co-Pt alloy with a composition very similar in the four samples studied. Its composition is close to a $\mathrm{Co}_{0.5} \mathrm{Pt}_{0.5}$ alloy, according to the Co and Pt coordination and to the Co-Co, Co-Pt and Pt-Pt distances found by EXAFS analysis of the samples. In addition to this alloy, there might be a remnant of the original Co cluster formed on the alumina matrix. Thus the $\mathrm{Co}_{0.5} \mathrm{Pt}_{0.5}$ alloy is probably surrounding this Co rich core. In most of the samples, there is an excess of metallic Pt that 


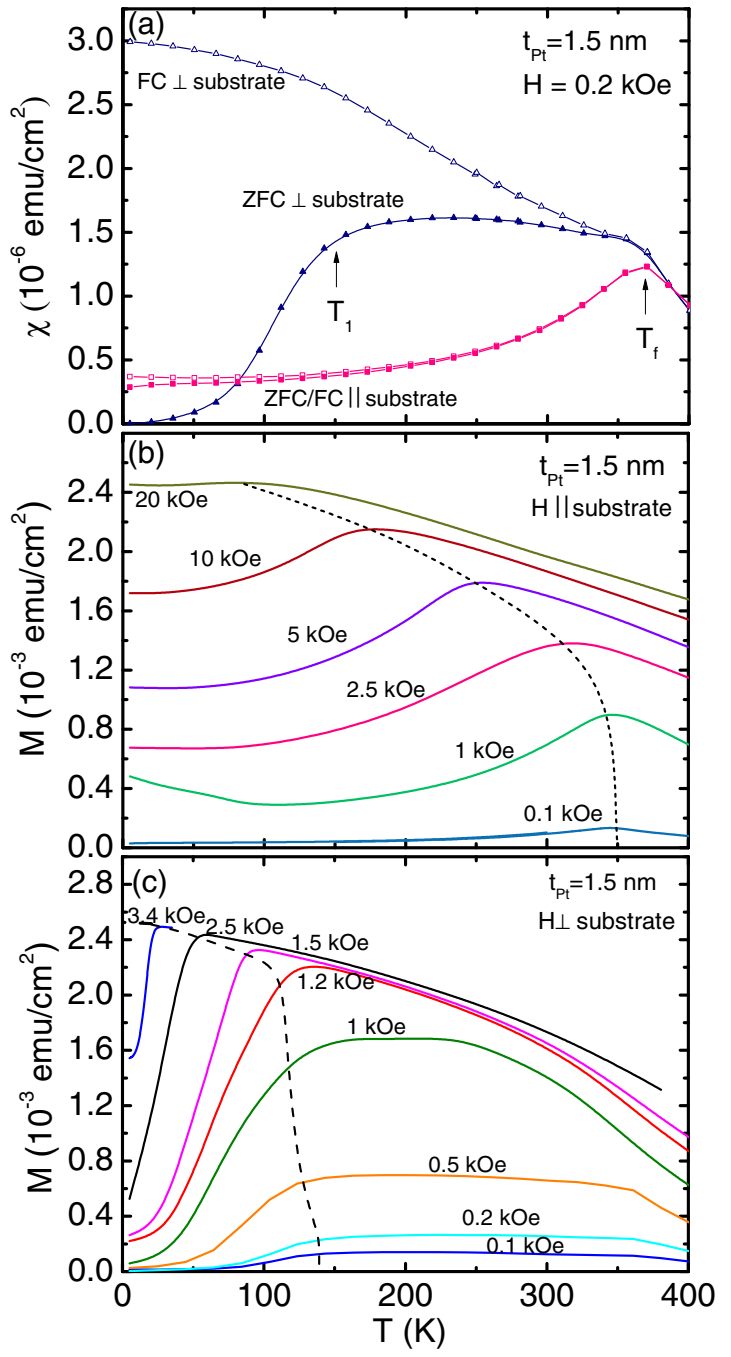

FIG. 7. (Color online) Direct current (dc) susceptibility curves measured under an applied field of $H_{\mathrm{dc}}=200$ Oe perpendicular and parallel to the substrate plane for the Co-Pt sample with $t_{\mathrm{Pt}}=1.5 \mathrm{~nm}$. (b) $M(T)$ curves measured for the same sample at various fields applied in the parallel direction. $(\cdots) M\left(T_{f}, H_{\mathrm{dc}}\right)$, superparamagnetic-soft ferromagnetic phase separation line. (c) $M(T)$ with the field applied in the perpendicular direction. (- - -) $M\left(T_{1}, H_{\mathrm{dc}}\right)$ soft-hard ferromagnetic phase separation line. Values of $H_{\mathrm{dc}}$ are indicated beside each curve.

crystallizes as an $f c c$ structure, filling the interparticle spaces, in agreement to what was observed by HRTEM. The presence of small Pt clusters in the $\mathrm{Al}_{2} \mathrm{O}_{3}$ matrix is also observed from the HRTEM images of the latter two samples. The structure of such Pt clusters is that of metallic fcc Pt, which contributes to the signal of metallic Pt identified in the Pt $L_{3}$-edge EXAFS.

\section{MAGNETIC PROPERTIES: SQUID-BASED MAGNETOMETRY}

The dc susceptibility curves measured with a bias field of $H_{\mathrm{dc}}=0.2 \mathrm{kOe}$, in both orthogonal configurations, for a representative sample (that with $t_{\mathrm{Pt}}=1.5 \mathrm{~nm}$ ) are plotted in Fig. 7(a). The shape of the curves is very different to that found for the bare Co NPs of the same $t_{\text {Co }}=0.7 \mathrm{~nm}$, which shows
TABLE III. Summary of the parameters deduced from the magnetic measurements on Co-Pt granular multilayers. Coercive fields $H_{\mathrm{C} \perp}$ and $H_{\mathrm{C} \|}$, saturation magnetization $M_{\mathrm{S}}$ and effective anisotropy constant $K_{\text {eff }}$ estimated from data measured at $T=5 \mathrm{~K}$.

\begin{tabular}{lccccccc}
\hline \hline $\begin{array}{l}t_{\mathrm{Pt}} \\
(\mathrm{nm})\end{array}$ & $\begin{array}{c}T_{1} \\
(\mathrm{~K})\end{array}$ & $\begin{array}{c}T_{f} \\
(\mathrm{~K})\end{array}$ & $\begin{array}{c}T_{\mathrm{Hc}} \\
(\mathrm{K})\end{array}$ & $\begin{array}{c}H_{\mathrm{C} \perp} \\
(\mathrm{kOe})\end{array}$ & $\begin{array}{c}H_{\mathrm{C} \|} \\
(\mathrm{kOe})\end{array}$ & $\begin{array}{c}M_{\mathrm{S}} \\
\left(10^{-3}\right.\end{array}$ & $\begin{array}{c}K_{\mathrm{eff}} \\
\left.\mathrm{emu} / \mathrm{cm}^{2}\right)\end{array}$ \\
\hline 0.6 & $\sim 110$ & 310 & $\sim 100$ & 2.3 & 0.81 & 2.53 & 4.6 \\
1.5 & $\sim 140$ & 365 & $\sim 200$ & 4.0 & 0.85 & 2.75 & 4.8 \\
4.5 & $\sim 140$ & 340 & $\sim 200$ & 3.0 & 0.46 & 2.91 & 2.4 \\
6.0 & $\sim 140$ & 296 & $\sim 200$ & 1.9 & 0.15 & 2.64 & 0.9 \\
\hline \hline
\end{tabular}

the typical ZFC-FC curve of a superparamagnetic system with a blocking temperature $T_{B}=27 \mathrm{~K}$ [2].

In the case of the Co-Pt granular films, the ZFC-FC curves measured in the perpendicular configuration show no overlapping as the temperature increases till it reaches the freezing temperature $T_{f}=310,365,340$, and $296 \mathrm{~K}$ for the samples with $t_{\mathrm{Pt}}=0.6,1.5,4.5$, and $6.0 \mathrm{~nm}$, respectively. In fact, three temperature regions are well distinguished from the ZFC curve, namely: (i) an increase of the magnetization with temperature until $T_{1}$ for the four samples in the series, which corresponds to the first shoulder observed in the curve in Fig. 7(a); (ii) a plateau between $T_{1}$ and $T_{f}$; and (iii) a decrease in the magnetization for temperatures above $T_{f}$ where both ZFC and FC curves merge following a Curie-Weiss law. Values of $T_{1}$ and $T_{f}$ identified for each sample are listed on Table III.

In contrast, the ZFC and FC curves measured in the parallel direction overlap even for fields as high as $50 \mathrm{kOe}$, showing a maximum at $T_{f}$. In all samples, increasing of the external applied field $H_{\mathrm{dc}}$ shifts $T_{f}$ to lower temperatures till the maximum is flattened and all the particles in the film become fully polarized along $H_{\mathrm{dc}}$, as observed in Fig. 7(b) for the sample with $t_{\mathrm{Pt}}=1.5 \mathrm{~nm}$. The dependence of $T_{f}$ with $H_{\mathrm{dc}}$ is a monotonously decreasing function $M\left(T_{f}, H_{\mathrm{dc}}\right)$, as shown in Fig. 7(b).

ZFC curves measured under different $H_{\mathrm{dc}}$, in the perpendicular direction, for the same sample with $t_{\mathrm{Pt}}=1.5 \mathrm{~nm}$ are depicted in Fig. 7(c). For increasing bias field, the threshold temperature $T_{1}$ decreases, tending to zero for $H_{\mathrm{dc}} \sim 4 \mathrm{kOe}$. $T_{f}$ also decreases with increasing $H_{\mathrm{dc}}$, so that the temperature limits of the plateau above $T_{1}$ becomes narrower and collapses with the $M\left(T_{1}, H_{\mathrm{dc}}\right)$ curve.

The same cusp at $T_{f}$ is observed in the real part of the ac susceptibility data, measured with a 4 Oe exciting field, both in the perpendicular and the parallel configuration, as depicted in Fig. 8. Between 1 and $480 \mathrm{~Hz}$, these data show no dependence on frequency and no distinctive features are observed at $T_{1}$.

To conclude, the field dependence of $T_{1}$ and $T_{f}$ are best observed from the ZFC measured in the perpendicular and parallel configurations, thereby defining the magnetic phase diagram of the multilayer, as illustrated in Fig. 9. It consists of three magnetic regions, namely: hard ferromagnetic, soft ferromagnetic and superparamagnetic.

The magnetic behavior of the Co-Pt grains within each region is further understood from the variation of the magnetization with applied field. $M(H)$ curves were measured for all samples with both configurations at several 


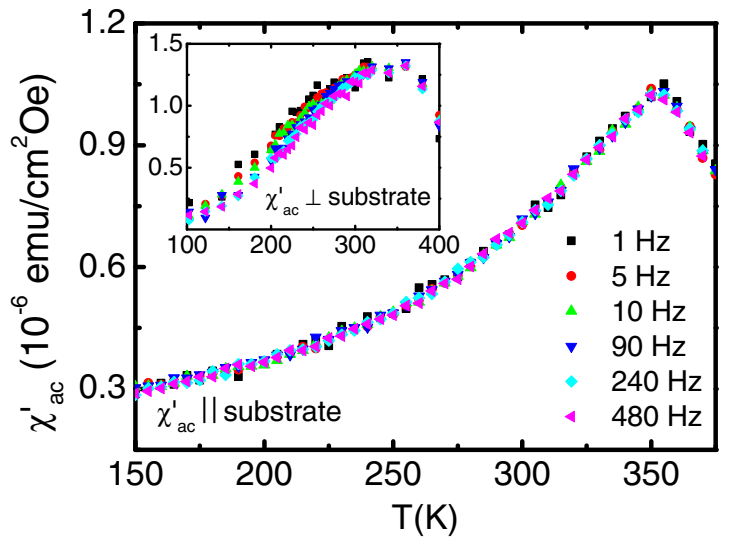

FIG. 8. (Color online) Real part of the ac susceptibility for the sample with $t_{\mathrm{Co}}=0.7 \mathrm{~nm}$ and $t_{\mathrm{Pt}}=1.5 \mathrm{~nm}$ measured with the applied ac field in the parallel configuration as a function of temperature using different excitation frequencies. (Inset) Analogous ac susceptibility curves measured for the same sample in the perpendicular configuration.

temperatures. Figure 10(a) shows the data recorded at $5 \mathrm{~K}$ (i.e., at $T<T_{1}$ ) for the sample with $t_{\mathrm{Pt}}=1.5 \mathrm{~nm}$. The magnetization is expressed per substrate area, as a usual practice in magnetic recording media and magnetic heterostructures. At low temperatures, the hysteresis curves measured in the perpendicular direction are rather square, which indicates the presence of a ferromagnetic hard component, with a quite high coercive field of $H_{\mathrm{C} \perp}=2.3,4.0,3.0$, and $1.9 \mathrm{kOe}$ for the samples with $t_{\mathrm{Pt}}=0.6,1.5,4.5$, and $6.0 \mathrm{~nm}$, respectively, at $T=5 \mathrm{~K}$. Such a ferromagnetic behavior implies that the Co-Pt particles in the film are strongly coupled.

In addition to the main reversal of the magnetization close to $H_{\mathrm{C} \perp}$, the hysteresis curves measured at low temperatures in the perpendicular direction have a slight step near $H=0$, which indicates that there is a magnetically soft component present in the sample [see arrow A on Fig. 10(a)].

The coercive field measured in the perpendicular direction $\left(H_{\mathrm{C} \perp}\right)$ decreases with temperature till it disappears at about $T_{\mathrm{Hc}} \sim 200 \mathrm{~K}$ for most of the samples in the series $\left(T_{\mathrm{Hc}} \sim 100 \mathrm{~K}\right.$

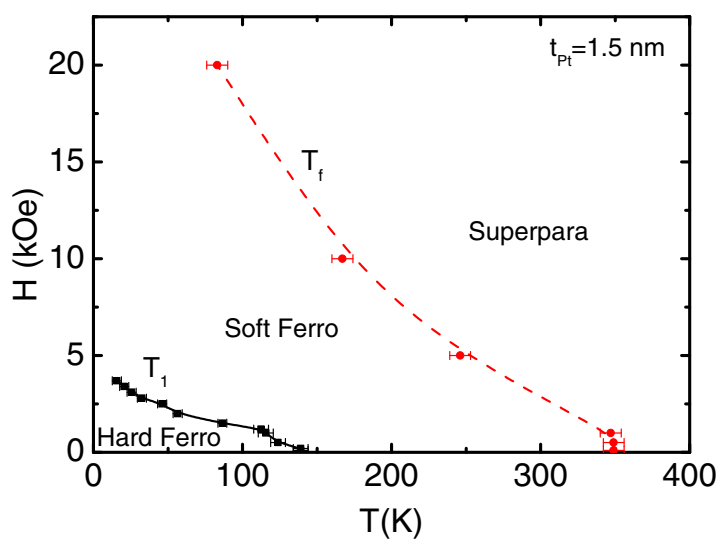

FIG. 9. (Color online) Magnetic phase diagram of the Co-Pt sample with $t_{\mathrm{Co}}=0.7 \mathrm{~nm}, t_{\mathrm{Pt}}=1.5 \mathrm{~nm}$ and $N=25$, as deduced from $M\left(T, H_{\mathrm{dc}}\right)$ measurements.
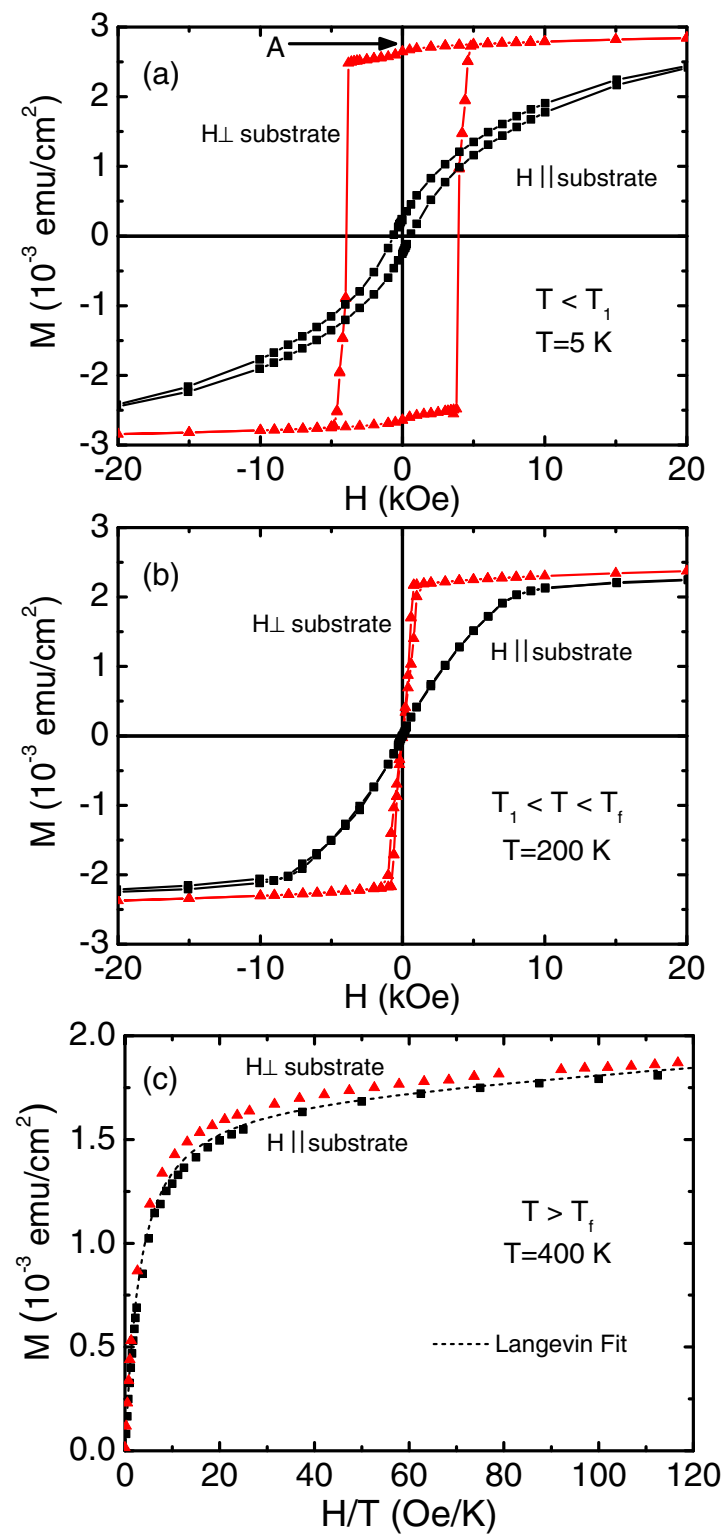

FIG. 10. (Color online) Magnetization curves measured for the sample with $t_{\mathrm{Co}}=0.7 \mathrm{~nm}$ and $t_{\mathrm{Pt}}=1.5 \mathrm{~nm}$, with $H_{\mathrm{dc}}$ applied perpendicular ( $\Delta$ ) and parallel (ם) to the substrate: (a) $T=5$, (b) 200 , and (c) $400 \mathrm{~K}$. The dashed line in (c) is the fitted Langevin curve with $\langle D\rangle=3.5 \mathrm{~nm}$ and $\sigma=0.4 \mathrm{~nm}$.

for the sample with $t_{\mathrm{Pt}}=0.6 \mathrm{~nm}$ ), as shown in Figs. 10(b) and 11. $H_{\mathrm{C} \perp}$ also decreases as $t_{\mathrm{Pt}}$ in the sample increases, except for some temperatures near $100 \mathrm{~K}$, where $H_{\mathrm{C} \perp}$ measured for the sample with $t_{\mathrm{Pt}}=4.5 \mathrm{~nm}$ is higher than that of the sample with $t_{\mathrm{Pt}}=1.5 \mathrm{~nm}$. This, in principle, unusual behavior has also been reported for $\mathrm{Co} / \mathrm{Pt}$ multilayers where an oscillatory dependence of $H_{\mathrm{C}}$ with $t_{\mathrm{Pt}}$ and $N$ is observed, and has been ascribed to RKKY interactions between the Co layers through Pt [45].

In the direction parallel to the substrate plane, the coercive field is much lower, being $0.81,0.85,0.46$, and $0.15 \mathrm{kOe}$ for samples with $t_{\mathrm{Pt}}=0.6,1.5,4.5$, and $6.0 \mathrm{~nm}$, respectively, at $5 \mathrm{~K}$ [see Fig. 10(a)]. Such differences between the magnetization curves measured in orthogonal directions (with the bias field 


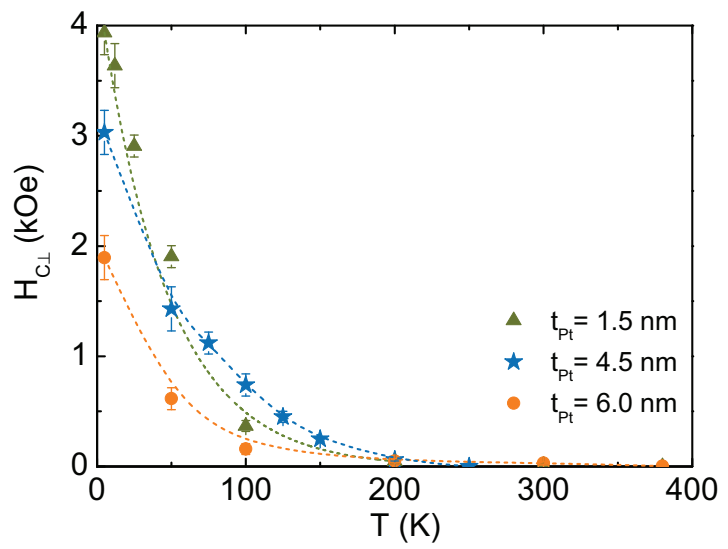

FIG. 11. (Color online) Temperature dependence of the coercive field measured with $H_{\mathrm{dc}}$ applied perpendicular to the substrate plane for the samples with $t_{\mathrm{Co}}=0.7 \mathrm{~nm}$ and (a) ( $\Delta$ ) $t_{\mathrm{Pt}}=1.5$, (b) ( $\star$ ) 4.5 , and (b) (•) $6.0 \mathrm{~nm}$.

applied parallel and perpendicular to the substrate plane) evidence the presence of perpendicular magnetic anisotropy (PMA) in the Co-Pt samples. The easy magnetization direction has been identified to be the one perpendicular to the substrate.

The magnetic anisotropy of the Co-Pt samples can be quantified by analysis of the hysteresis loops measured in the two orthogonal configurations. The magnetic anisotropy energy can be estimated from the area enclosed by the two curves (the parallel and perpendicular configurations), in the first quadrant of the $M(H)$ curve. This area is calculated after averaging the two branches of the hysteresis loop for each configuration [8]. The resulting curves for a representative sample (the one with $t_{\mathrm{Pt}}=1.5 \mathrm{~nm}$ ) are plotted in Fig. 12. This area yields the quantity $K_{\text {eff }} t$, with $K_{\text {eff }}$ the effective anisotropy constant and $t$ the film thickness. We have found values of $K_{\text {eff }} t=10(1), 12(1), 13(1)$, and 6(1) $\mathrm{erg} / \mathrm{cm}^{2}$ at $T=5 \mathrm{~K}$ for the samples with $t_{\mathrm{Pt}}=0.6,1.5,4.5$, and $6.0 \mathrm{~nm}$, respectively, at $5 \mathrm{~K}$. According to the morphological study described in Sec. IV A, each Co-Pt layer in our films is about 2.3-, 2.5-,

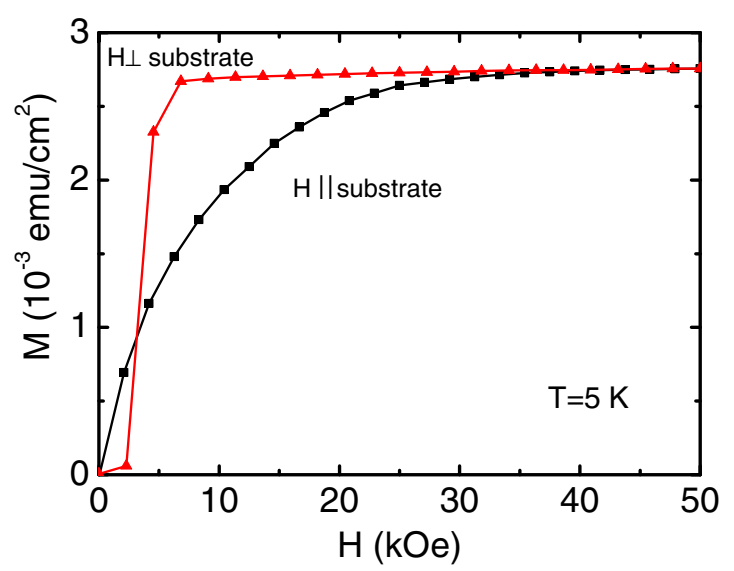

FIG. 12. (Color online) Average of the two branches of the hysteresis curves measured for the sample with $t_{\mathrm{Co}}=0.7 \mathrm{~nm}$ and $t_{\mathrm{Pt}}=1.5 \mathrm{~nm}$, with $H_{\mathrm{dc}}$ applied perpendicular ( $\left.\boldsymbol{\Delta}\right)$ and parallel (ם) to the substrate at $T=5 \mathrm{~K}$.
5.5-, and 6.7-nm thick, for the samples with $t_{\mathrm{Pt}}=0.6,1.5,4.5$, and $6.0 \mathrm{~nm}$, respectively. Then, $K_{\text {eff }}$ results in values of 4.6, 4.8, 2.4, and $0.9 \times 10^{7} \mathrm{erg} / \mathrm{cm}^{3}$ at $T=5 \mathrm{~K}$ for the samples with $t_{\mathrm{Pt}}=0.6,1.5,4.5$, and $6.0 \mathrm{~nm}$, respectively. $K_{\text {eff }}$ decreases with the increase in $t_{\mathrm{Pt}}$, following the same trend as the $H_{\mathrm{C} \perp}$ in these samples.

Above $T_{f}$ the $M(H)$ curves measured in both configurations have the characteristic superparamagnetic behavior, as shown in Fig. 10(c). These curves can be fitted to a Langevin curve for Co-Pt particle average magnetization, plus a paramagnetic contribution caused by loose Co atoms, following the expression:

$$
M(H)=\mu\left[\operatorname{coth}\left(\frac{\mu H}{k_{B} T}\right)-\frac{k_{B} T}{\mu H}\right]+\chi_{o} H .
$$

From the fit, one obtains the average diameter of the magnetic grain contributing to this magnetization curve. It results in an average diameter of $\langle D\rangle=3.5 \mathrm{~nm}$ and $\sigma=0.4 \mathrm{~nm}$

\section{MAGNETIC PROPERTIES: ANOMALOUS HALL EFFECT MAGNETOMETRY}

Measurements of Hall voltage in magnetic materials have an additional contribution proportional to their magnetization, called the extraordinary or anomalous Hall effect (AHE). The AHE arises from spin dependent scattering mechanisms, so that correlation between the AHE and the magnetization enables the use of the Hall voltage measurement for the study of magnetic properties of materials in conditions hardly accessible by other techniques [46]. Indeed, it is a method frequently used to study the magnetic properties of thin films exhibiting PMA. Measurements of AHE in ferromagnetic granular systems has demonstrated its sensitivity to the local magnetic moments of individual nanoparticles [47], so that hysteresis is developed in the Hall voltage $\left(V_{H}\right)$ versus applied field curve below the blocking temperature of magnetic particles. Thus, by measuring the variation of $V_{H}$ with the magnetic field applied in the direction perpendicular to the substrate of our Co-Pt granular samples, hysteresis loops comparable with those of SQUID magnetometry are obtained.

In Fig. 13, we have plotted those hysteresis loops obtained for the two Co-Pt samples at $T=5 \mathrm{~K}$. These curves show a remarkable squareness and the coercive field coincides well with the one obtained by SQUID magnetometry (see Fig. 10). However, the soft component observed in the curves measured in the SQUID magnetometer is not detected by AHE, which instead seems to be very sensitive to the hard component. We may understand these AHE measurements as due to the scattering of the charge carriers by the Co-Pt magnetic grains, which are embedded in a conducting matrix (the excess of Pt in the interparticle spaces). The AHE voltage is then proportional to magnetic moment of individual magnetic clusters averaged over the volume of the sample. Similar results have been obtained by AHE measurements of fcc Co clusters prepared by LECBD, embedded in a Pt matrix $[46,47]$. The $H_{\mathrm{C} \perp}$ values and their temperature dependence obtained by this method in our Co-Pt granular films perfectly match those identified by SQUID magnetometry in Fig. 11, as observed in the direct 


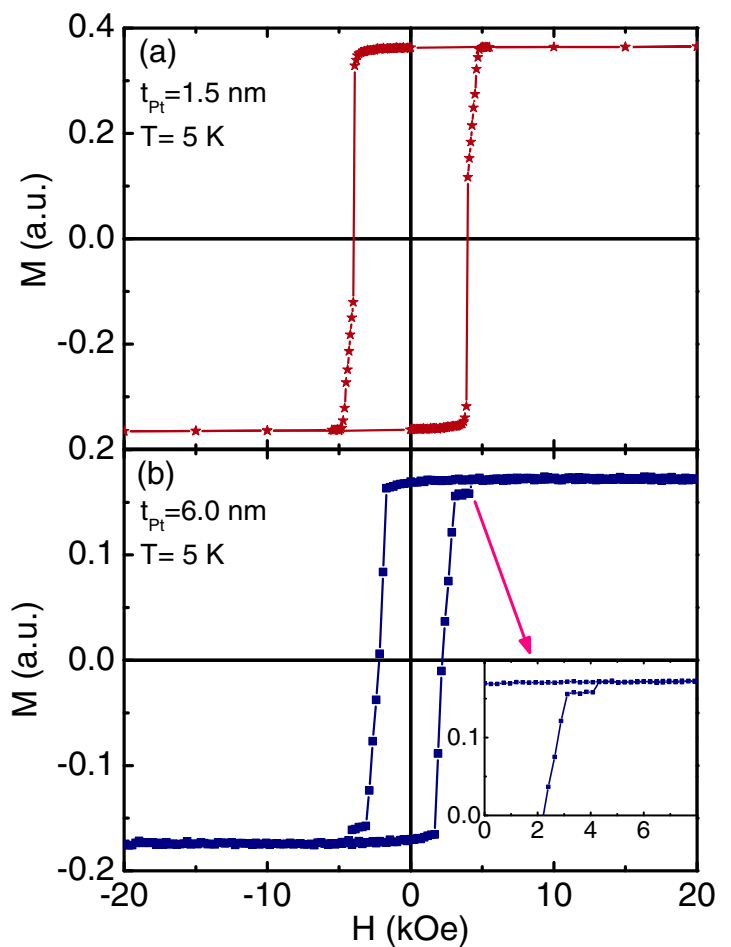

FIG. 13. (Color online) AHE hysteresis loops measured at $T=$ $5 \mathrm{~K}$ and $H_{\mathrm{dc}}$ perpendicular to the substrate for the samples with $t_{\mathrm{Co}}=0.7 \mathrm{~nm}$ and (a) $t_{\mathrm{Pt}}=1.5$ and (b) $6.0 \mathrm{~nm}$. Inset in (b): details of the first quadrant of the hysteresis loop in (b).

comparison of $H_{\mathrm{C} \perp}$ versus $T$ for both techniques depicted in Fig. 14.

AHE measurements also allow to examine the magnetization reversal mechanisms in our granular films. The inset of Fig. 13(b) reveals details of the switching of magnetization in the sample with $t_{\mathrm{Pt}}=6.0 \mathrm{~nm}$, which was also observed in the hysteresis curve measured in the perpendicular configuration with the SQUID in this sample (data not shown). In the first and third quadrants, the magnetization reversal in this sample apparently proceeds layer by layer. The last layer

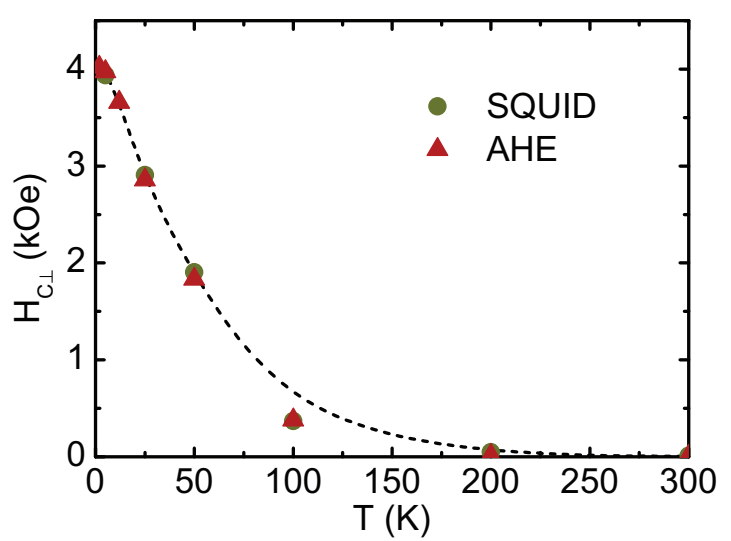

FIG. 14. (Color online) Comparison of the $H_{\mathrm{C}}$ vs $T$ obtained

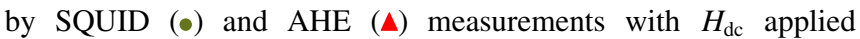
perpendicular to the substrate plane of the sample with $t_{\mathrm{Co}}=0.7 \mathrm{~nm}$ and $t_{\mathrm{Pt}}=1.5 \mathrm{~nm}$. Dashed line: guide to the eye. magnetization reversal involves a variation of $\Delta M_{s} / M_{s}=$ $1 / 12.1$, where $M_{s}$ is the saturation moment (measured by SQUID magnetometry). Indeed, if each layer contributes $M_{1}=M_{s} / 25$ to the total magnetization, the reversal of the magnetization of one layer will imply a moment difference of twice this quantity.

\section{MAGNETIC PROPERTIES: X-RAY MAGNETIC CIRCULAR DICHROISM MAGNETOMETRY}

\section{A. Co $K$-edge results}

XANES and XMCD at the Co $K$ edge probes the $4 p$ empty states of the Co atom in the sample. Analysis of the XANES spectra recorded at this edge on these Co-Pt samples was described in section IV B. Evidences of Co $4 p-3 d$ and Co $3 d-\mathrm{Pt} 5 d$ hybridization in the Co-Pt granular samples and electronic transfer from Pt $5 d$ to Co orbitals were then described. The detection of a nonzero XMCD signal at the Co $K$ edge reflects the magnetic polarization of the Co $4 p$ band caused by the intra-atomic exchange interaction with the $3 d$ band. The XMCD signal recorded at this edge for the Co-Pt granular samples is shown in Fig. 15, normalized to the absorption jump obtained in the Co $K$ XAS spectrum after background removal. It has the same sign as the Co $K$ XMCD reported in Ref. [48], which results in an antiferromagnetic coupling between $4 p$ and $3 d$ spins in Co

The shape of XMCD signal in Fig. 15 is very similar to the one reported for ion irradiated Co-Pt films in Ref. [49]. In those Co-Pt films, changes in the electronic structure of $\mathrm{Co}$ atoms related to the formation of $\mathrm{Co}_{x} \mathrm{Pt}_{1-x}$ alloys induced by ion irradiation were observed. Striking similarities between the XMCD spectra of the Co-Pt films and a $\mathrm{Co}_{0.5} \mathrm{Pt}_{0.5} L 1_{0}$ thinfilm reference sample were shown, in terms of characteristic

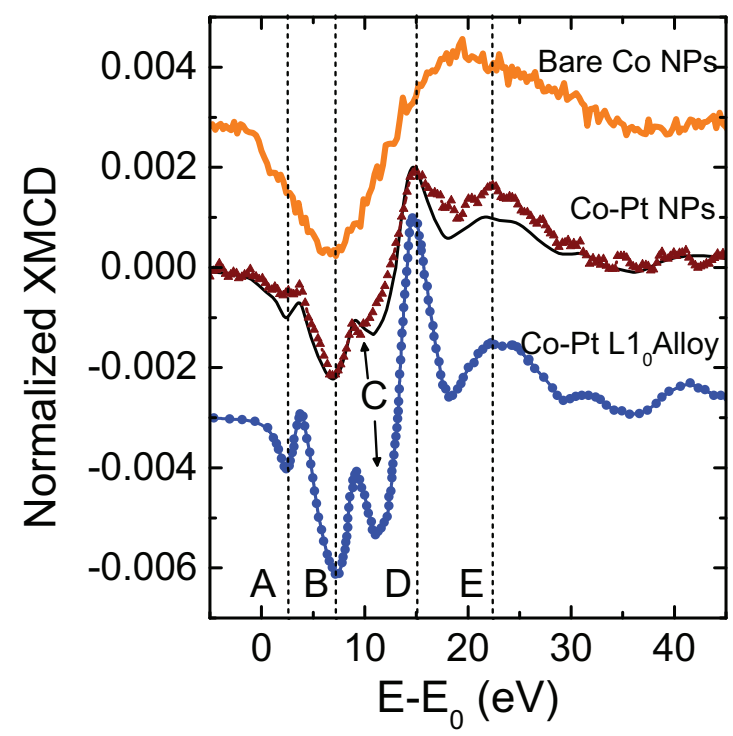

FIG. 15. (Color online) Co $K$-edge XMCD signal measured in the Co-Pt granular film $(\boldsymbol{\Lambda})$ with $t_{\mathrm{Co}}=0.7 \mathrm{~nm}$ and $t_{\mathrm{Pt}}=1.5 \mathrm{~nm}$ and comparison to that of a Co-Pt $L 1_{0}$ alloy (•) from Ref. [49] and for bare Co NPs (_) with the same $t_{\mathrm{Co}}$. Solid line corresponds to a fit of the XMCD for the Co-Pt granular film, as a linear combination of the other two curves. Curves are shifted vertically for the sake of clarity. 
peaks at selected energies. This allowed to interpret the XMCD spectrum for the irradiated Co-Pt film as the superposition of pure Co and $L 1_{0} \mathrm{Co}_{0.5} \mathrm{Pt}_{0.5}$ alloy contributions.

Similarly, in our Co-Pt samples we may state that the Co $K$-edge XMCD signal that we observe is a combination of these two phases. This is illustrated in Fig. 15, where a direct comparison of the XMCD recorded for our Co-Pt granular films with that of a Co-Pt $L 1_{0}$ alloy (from Ref. [49]) and for bare Co NPs with the same $t_{\mathrm{Co}}$ is performed. Features of the metallic Co XMCD, such as the peak denoted as B in the figure, are identified in the three curves. Peaks A, C, D, and E are common in both the Co-Pt alloy and our Co-Pt granular films, supporting the presence of this alloy in our Co-Pt samples.

Indeed, we have been able to fit the experimental XMCD spectrum obtained at the Co $K$ edge of the Co-Pt granular films (GF) as a linear combination of two empirical reference contributions: the experimental XMCD signal from Co bare NPs (of the same nominal size than those studied in this paper) and the $\mathrm{XMCD} \mathrm{Co}_{0.5} \mathrm{Pt}_{0.5} \mathrm{~L}_{0}$ thin-film reference sample from Ref. [49]. To do so, we have fitted both references with a sum of a number of lorentzian curves sufficient to reproduce the spectral features in detail. Later on, the experimental data have been fitted to a linear combination of the two analytical reference functions

$$
\mathrm{XMCD}_{\exp }^{\mathrm{GF}}(E)=a \mathrm{XMCD}_{\mathrm{L}_{0}}(E)+b \mathrm{XMCD}_{\mathrm{Co}}(E) .
$$

The best fit to the Co-Pt data, shown as a solid line in Fig. 15, is obtained with $a=0.58(2)$ and $b=0.42$ (3), suggesting that approx. $60 \%$ of the cobalt initially deposited on the films alloys with Pt upon its deposition. From this comparison one concludes that peaks A, C, and D are characteristic of the $L 1_{0}$ Co-Pt alloy.

To dispel any question on the possibility of having the chemically disordered $A 1 \mathrm{Co}_{0.5} \mathrm{Pt}_{0.5}$ phase instead, we have calculated the Co $K$-edge XMCD signal of the two phases ( $L 1_{0}$ and $\left.A 1\right)$ and compared to the experimental data for $L 1_{0}$ (see Fig. 16).

The absorption cross sections were calculated selfconsistently with the fully relativistic FDMNES code [50,51] in the framework of multiple scattering theory within the muffin tin approximation. The structural and crystallographic

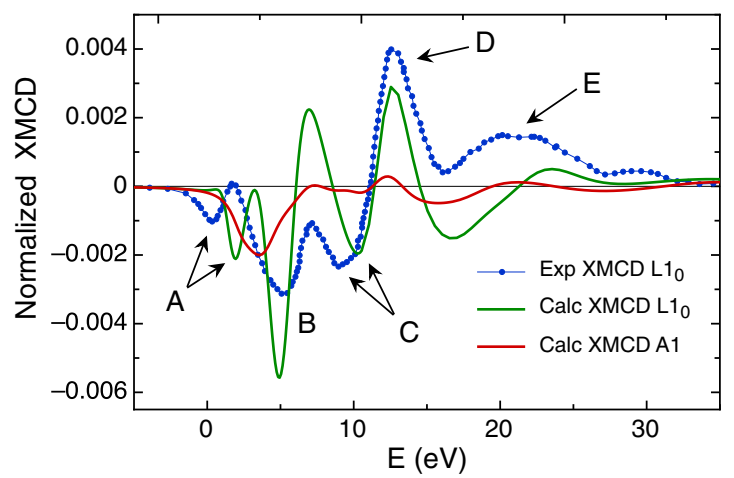

FIG. 16. (Color online) (॰) Experimental data from Ref. [49] and (-) calculated XMCD signal at the Co $K$ edge for a Co-Pt $L 1_{0}$ alloy. (-) The calculated XMCD for a $A 1$ Co-Pt alloy is also shown for comparison. information of the $L 1_{0} \mathrm{Co}_{0.5} \mathrm{Pt}_{0.5}$ alloy system used to define the position of the absorbing and scattering atoms in the calculations is the one previously described in Sec. IVB. The $A 1$ phase has been calculated by defining six " $A 1$ volumes" of $1.5 \mathrm{~nm}$ diameter, formed by 140 atoms in a fcc structure according to crystallographic information found in the ICSD [38]. Each atom of the $A 1$ volume is randomly attributed to Co or Pt with a ratio of 1:1. Generating a set of such 140 atoms-particles instead of a single, larger virtual $A 1$ volume is computationally beneficial: the self-consistent FDMNES calculation [51] on a larger chemically disordered volume yields impracticably long times and high allocated memory.

A cluster of 7- $\AA$ radius and a Fermi level of about $7701 \mathrm{eV}$ were used in both, $L 1_{0}$ and $A 1$ calculations. To avoid spurious "surface effects" in the virtual $A 1$ volumes, we selected absorbing Co atoms lying within the inner part of the particle, such that the whole $7 \AA$ FDMNES cluster around the absorbing atom fits inside the $A 1$ volume. The convolution of the results obtained for 24 randomly chosen Co atoms (four atoms from each different $A 1$ volume) is shown in Fig. 16.

In the $A 1$ phase, peaks $\mathrm{A}$ and $\mathrm{B}$ appear to be convoluted into a single, broader negative feature. At higher energies, peaks $\mathrm{C}, \mathrm{D}$, and $\mathrm{E}$ are strongly reduced in intensity, if not directly absent. This is expected for the sum of the oscillating $\mathrm{XMCD}$ from every Co atom, slightly shifted in energy by local chemical disorder. However, the lack of peak A is predicted in the XMCD signal of the $24 \mathrm{Co}$ absorbing atoms computed in a disordered environment. Conversely, its presence may be linked to the particular $L 1_{0}$ order. It is not possible to describe our experimental data in the Co-Pt granular films by means of a linear combination as Eq. (2) including only the XMCD signals of the $A 1$ phase and that of pure Co. In particular, features $\mathrm{A}$ and $\mathrm{D}$ would be completely missing.

Concluding, XMCD at the Co $K$ edge manifests the existence of both metallic Co and a $L 1_{0} \mathrm{Co}_{0.5} \mathrm{Pt}_{0.5}$ alloy in our Co-Pt granular samples, ruling out the presence of a significant amount of $A 1$ phase, thus supporting our EXAFS results (see Sec. IV B).

\section{Co $L_{2,3}$-edges results}

$\mathrm{XMCD}$ measurements at the Co $L_{2,3}$ edges as a function of the incident angle $\left(\theta=0^{\circ}\right.$ and $60^{\circ}$, with respect to the sample surface normal) have been performed, looking for anisotropy of the orbital moment. Figure 17(a) shows the Co $L_{2,3}$ XAS recorded at normal incidence, and Fig. 17(b) the XMCD signal obtained for each angle. The XMCD signal has been normalized to the XAS spectrum in high-energy limit, after background subtraction. We observe a clear angle dependence of the XMCD, which is also reflected in the variation of their integrated intensity [shown in Fig. 17(b)]. The angle dependent XMCD reveals the PMA in the film since its intensity, proportional to the magnetization component parallel to the x-ray beam, is larger for the normal incidence than that at $\theta=60^{\circ}$. These results are similar to the angle dependent Co $L_{2,3}$ XMCD observed for ion irradiated Co-Pt films with PMA in Ref. [32].

The anisotropy of the orbital moment is analyzed from the estimation of the in-plane and out-of-plane components of 


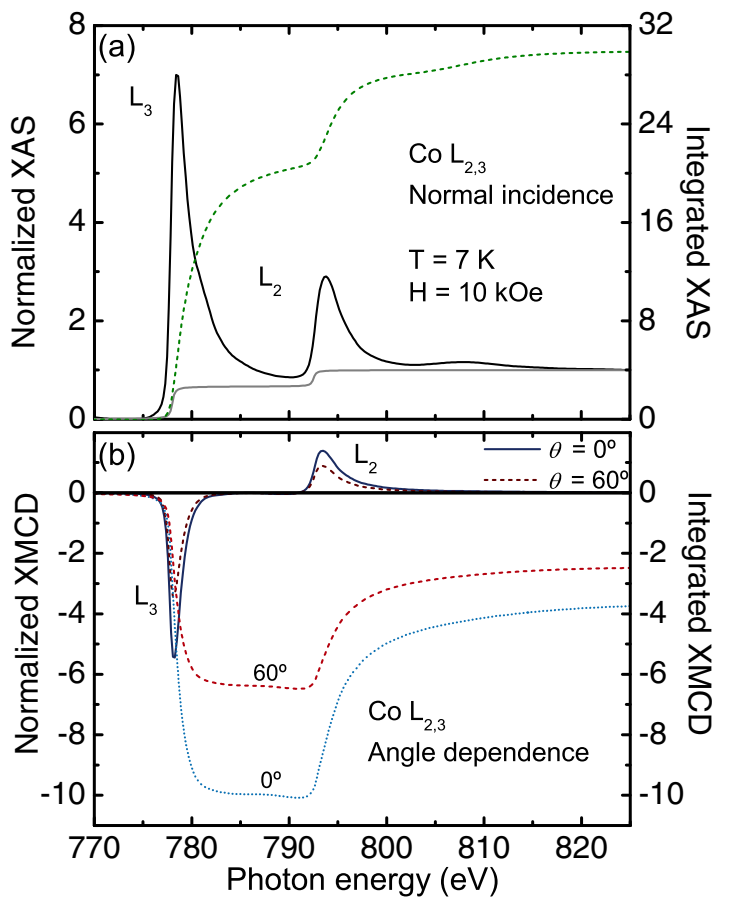

FIG. 17. (Color online) Co $L_{2,3}$-edge spectra of the $t_{\mathrm{Co}}=0.7 \mathrm{~nm}$ and $t_{\mathrm{Pt}}=1.5 \mathrm{~nm}$ sample. (a) (-) Normalized XAS spectra mean curve measured at normal incidence. (_) The double-step function used is also indicated. (_ _) Integrated white line. (b) Angle dependent normalized XMCD spectra: (_) normal incidence and (_ _) $60^{\circ}$. The integrated area of each XMCD signal is also plotted.

the orbital magnetic moment, $m_{\mathrm{L}}^{\|}$and $m_{\mathrm{L}}^{\perp}$, respectively, from the angle-dependent XMCD data. The orbital moment at each measured angle $m_{\mathrm{L}}^{\theta}$, as obtained from the magneto-optical sum rule [52], is expressed as

$$
m_{\mathrm{L}}^{\theta}=m_{\mathrm{L}}^{\|} \sin ^{2} \theta+m_{\mathrm{L}}^{\perp} \cos ^{2} \theta .
$$

However, in order to apply the magneto-optical sum rules $[52,53]$ to the XMCD signal in Fig. 17(b), the number of unoccupied states in the $3 d$ band for Co $n_{h}$ is required in the calculations. Values of $n_{h}$ at the Co $3 d$ band reported in the literature are, for example, $n_{h}=2.49$ holes for metallic Co [54] and $n_{h}=2.628$ for $\mathrm{Co}_{0.5} \mathrm{Pt}_{0.5} L 1_{0}$ alloy films [27]. Let us recall that the Co atoms in our Co-Pt granular samples are found to be in these two different environments (metallic Co and $\mathrm{Co}_{0.5} \mathrm{Pt}_{0.5} L 1_{0}$ alloy), but we are not able to quantify each of them. Therefore the right number of $3 d$ holes in Co atoms of our Co-Pt samples is unknown. Thus we have expressed the orbital moment as per $3 d$ hole in the Co atoms. In addition, given the highly anisotropic character of the Co-Pt granular film, the integrated $3 d$ absorption white line $r$ needs to be corrected for the angular dependence of the absorption intensity, which we have done following the procedure of Ref. [55]. We obtain $m_{\mathrm{L}}^{\|} / n_{h}=0.05(1) \mu_{B}$ and $m_{\mathrm{L}}^{\perp} / n_{h}=0.08(1) \mu_{B}$ from the application of the orbital sum rule to the XMCD at the two incident angles $\left(\theta=0^{\circ}\right.$ and $\left.60^{\circ}\right)$.

From the spin sum rule [53], we derive an effective spin magnetic moment per $3 d$ hole of $m_{\text {Seff }} / n_{h}=0.77(1) \mu_{B}$, at $\theta=0^{\circ}$. This value is comparable to the $m_{\text {Seff }} / n_{h}=0.75 \mu_{B}$ per Co atom obtained for a $\mathrm{Co}_{0.5} \mathrm{Pt}_{0.5} L 1_{0}$ alloy film in Ref. [27]. In order to obtain $m_{\mathrm{S}} / n_{h}$, one has to take into account the contribution of the angular dependent magnetic dipole term $m_{\mathrm{D}}$ since the $\mathrm{Co}_{0.5} \mathrm{Pt}_{0.5} L 1_{0}$ structure is highly anisotropic [27]. The angular dependence of $m_{\text {Seff }}$ is analogous to that of $m_{\mathrm{L}}$ defined by Eq. (3). At the so-called "magic angle" $\left(57.3^{\circ}\right), m_{\mathrm{D}}$ vanishes so that $m_{\text {Seff }}=m_{\mathrm{S}}[27,56]$. Thus, by applying the spin sum rule to the XMCD signal obtained at the two measured angles $\left(\theta=0^{\circ}\right.$ and $\left.60^{\circ}\right)$ in our Co-Pt granular films, we have derived $m_{\text {Seff }} / n_{h}\left(57.3^{\circ}\right)=m_{\mathrm{S}} / n_{h}=0.51(1) \mu_{B}$. Finally, at $\theta=0^{\circ} m_{\mathrm{L}} / m_{\text {Seff }}=0.11(1)$ and $m_{\mathrm{L}} / m_{\mathrm{S}}=0.16(1)$, being of the same order of that for a $\mathrm{Co}_{0.5} \mathrm{Pt}_{0.5} L 1_{0}$ alloy film $\left(m_{\mathrm{L}} / m_{\mathrm{S}}=0.15\right)[27]$.

XMCD measurements at the Co $L_{2,3}$ edges on $\mathrm{Co}_{x} \mathrm{Pt}_{1-x}$ particles prepared by vapor deposition have been recently reported [57,58]. They find $m_{\mathrm{L}} / m_{\mathrm{S}}$ ranging between 0.10 and 0.14 on particles of comparable size to ours, depending on the composition of the Co-Pt alloy present. Then, the $m_{\mathrm{L}} / m_{\mathrm{S}}$ (for $\theta=0^{\circ}$ ) is higher in our Co-Pt samples. With reference to other XMCD studies in Co-Pt systems, our $m_{\mathrm{L}} / m_{\mathrm{S}}$ in Co is also larger, as compared to $\mathrm{CoPt}_{3}$ films deposited on a substrate at $800 \mathrm{~K}\left(m_{\mathrm{L}} / m_{\mathrm{S}}=0.09\right)$ [26], and for annealed $\mathrm{Co}_{0.5} \mathrm{Pt}_{0.5}$ NPs with diameters of about $2.6 \mathrm{~nm}$ deposited on amorphous carbon matrices $\left(m_{\mathrm{L}} / m_{\mathrm{S}}=0.094\right)$ [10].

\section{B. Pt $L_{2,3}$-edge results}

The XMCD spectra measured at the Pt $L_{2,3}$ edges are shown in Fig. 18. The nonzero values of the XMCD signal reflects the polarization of the Pt by the magnetic Co. From an XMCD experiment at the Pt $L_{2,3}$ edges one obtains, through the sum

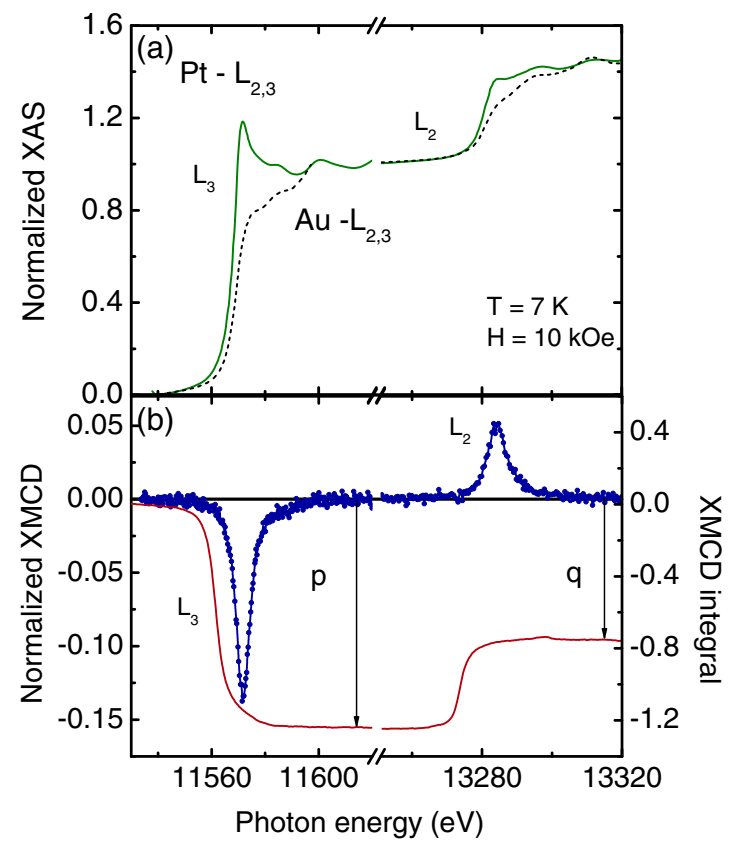

FIG. 18. (Color online) (-) Normalized XANES (a) and (•) $\mathrm{XMCD}$ (b) at the Pt $L_{2,3}$ edges in the Co-Pt sample with $t_{\mathrm{Co}}=0.7$ $\mathrm{nm}$ and $t_{\mathrm{Pt}}=1.5 \mathrm{~nm}$. (-) XMCD integrated area. (- -) The XANES spectrum of $\mathrm{Au} L_{2,3}$ edges is shown in (a) for comparison. The higher limit for the $p$ and $q$ integrals, used for the sum rules analysis, is given in (b). 
rules [52,53], the spin $m_{\mathrm{S}}$ and orbital $m_{\mathrm{L}}$ moments averaged over the whole sample, provided one knows by calculation or estimation the number of holes, $n_{h}$, existing involved in the $2 p \rightarrow 5 d$ excitations. For $\mathrm{Pt}$, we have derived $n_{h}$ from the white line difference with a Pt foil at the $L_{2,3}$-edge XANES, similarly to the procedure followed for XANES at the W $L_{2,3}$ edges for Co-W NPs in Ref. [59]. We have obtained $n_{h}=1.80(2)$ holes in the $5 d$ band, $m_{\mathrm{S}}=0.14(1) \mu_{B}, m_{\mathrm{L}}=$ $0.027(2) \mu_{B}$, and $m_{\mathrm{L}} / m_{\mathrm{S}}=0.19(1)$ for the Pt atoms in our Co-Pt granular films. We have assumed that the contribution of the dipolar term to the effective spin moment is negligible; indeed, $m_{\text {Seff }}$ in the Pt atoms is expected to be isotropic given the strong delocalization of the $5 d$ electrons, as described for similar Pt $L_{2,3}$ XMCD studies of Co-Pt systems [27].

The induced moment on Pt $m_{\text {tot }}=0.16(1) \mu_{B}$ is one order of magnitude larger than the moments induced on the $\mathrm{Cu}$, $\mathrm{Ag}$, or Au capping cases [6]. Obviously, the reason for this difference is the proximity of $\mathrm{Pt}$, with the $5 d^{9}$ band, to fulfill the Stoner criterion for ferromagnetism [60], instead of the nearly filled noble metal $n d^{10}$ bands $(n=3,4$, and 5 for $\mathrm{Cu}$, $\mathrm{Ag}$, or $\mathrm{Au}$, respectively).

However, it is important to point out that not all $\mathrm{Pt}$ atoms in the Co-Pt granular sample $\left(t_{\mathrm{Co}}=0.7 \mathrm{~nm}\right.$ and $\left.t_{\mathrm{Pt}}=1.5 \mathrm{~nm}\right)$ are alloyed or in contact with Co atoms, as described in the HRTEM and EXAFS measurements (see Sec. IV) performed in this sample. As a result, it is plausible that not all Pt atoms in the sample are polarized by Co. Only those polarized Pt atoms contribute to the $\mathrm{Pt} L_{2,3}$ XMCD signal, but all $\mathrm{Pt}$ atoms in the sample are reflected in the Pt $L_{2,3}$ XAS. Since the XMCD results are scaled to the Pt $L_{2,3}$ XAS spectra, the $m_{\mathrm{S}}$ and $m_{\mathrm{L}}$ results from Pt XMCD may be lower than the actual ones. The $m_{\mathrm{L}} / m_{\mathrm{S}}$ ratio depends only on the XMCD integral values, so it is not affected by the $\mathrm{Pt} L_{2,3}$ XAS scale and we may rely on that value.

Comparing to XMCD measurements at the Pt $L_{2,3}$ edges reported in the literature, the $m_{\mathrm{L}} / m_{\mathrm{S}}$ ratio in the $5 d$ band of $\mathrm{Pt}$ in our Co-Pt granular films is close to those values obtained for $\mathrm{CoPt}_{3}$ films in Ref. [26] $\left(m_{\mathrm{L}} / m_{\mathrm{S}}=0.18-0.26\right)$, but lower than those of $\mathrm{Co}_{0.5} \mathrm{Pt}_{0.5} L 1_{0}$ alloy films $\left(m_{\mathrm{L}} / m_{\mathrm{S}}=0.27\right)$ [27].

\section{Element-specific magnetic hysteresis measured by XMCD}

One important advantage of the selective magnetometry using the XMCD technique is the possibility of performing element-specific magnetic hysteresis measurements. It consists in recording the dichroic signal for a fixed energy while the applied magnetic field is varied, similarly to the magnetization hysteresis measurements with conventional magnetometers. In this way it is possible to evaluate the contribution of each atomic species to the magnetic properties of the material. We have performed $\operatorname{XMCD}(H)$ at the Co $K$ and $\mathrm{Pt} L_{3}$ edges on the same Co-Pt sample with $t_{\mathrm{Co}}=$ $0.7 \mathrm{~nm}$ and $t_{\mathrm{Pt}}=1.5 \mathrm{~nm}$. Measurements were done at the ESRF ID12 beamline, with the same technical details than in the conventional XMCD experiments described above.

Figure 19 shows the $\mathrm{XMCD}(H)$ measurements recorded from the XMCD spectra at the Co $K$ and $\mathrm{Pt} L_{3}$ edges in normal incidence at $12 \mathrm{~K}$. The energies at which $\mathrm{XMCD}(H)$ were collected were 7723.9 and $11571.5 \mathrm{eV}$ for Co $K$ and $\mathrm{Pt}$ $L_{3}$ edges, respectively. Notice that both hysteresis loops are

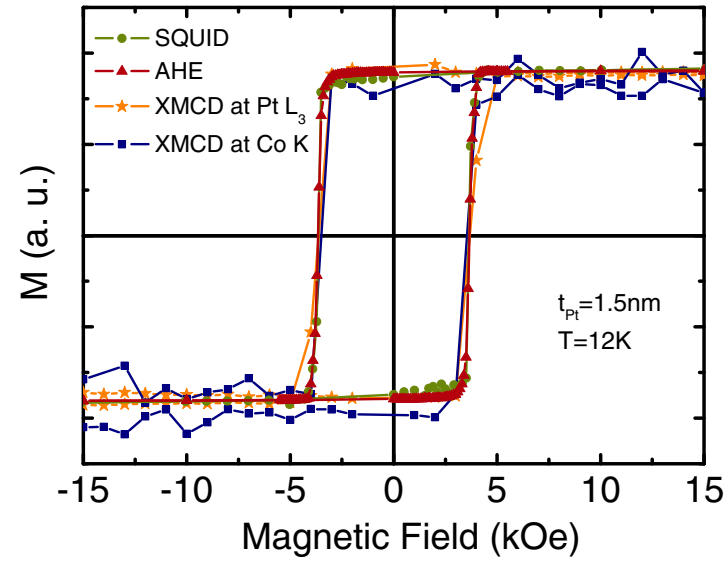

FIG. 19. (Color online) Comparison of the magnetic hysteresis loops measured at $T=12 \mathrm{~K}$ with different techniques in the Co-Pt granular sample with $t_{\mathrm{Co}}=0.7 \mathrm{~nm}$ and $t_{\mathrm{Pt}}=1.5 \mathrm{~nm}$ : (•) SQUID magnetometry, $(\boldsymbol{\Delta}) \mathrm{AHE},(\star) \mathrm{XMCD}$ at the $\mathrm{Pt} L_{3}$ edge, and (ם) $\mathrm{XMCD}$ at the Co $K$ edge.

squared, with a coercive field around $3.6 \mathrm{kOe}$ in both cases, which are consistent with those curves measured by SQUID (Sec. V) and AHE (Sec. VI) techniques for the same sample, perpendicular configuration and temperature, also included in Fig. 19 for the sake of a direct comparison. The " $M$ " axis has been scaled for all curves to coincide. The agreement in the $H_{\mathrm{C} \perp}$ values obtained by $\mathrm{XMCD}(H)$ is an indicative that $\mathrm{Co}$ and $\mathrm{Pt}$ atoms in the Co-Pt granular films are strongly magnetically coupled, as resulting from the Co-Pt alloy in the sample. Both $\mathrm{Co}$ and $\mathrm{Pt}$ atoms are responsible of the magnetic hard component measured in the sample with the magnetic field in the perpendicular configuration. No clear observation of the soft component has been detected in these measurements.

The decay of the $H_{\mathrm{C} \perp}$ values with increasing temperature has been also examined from the $\operatorname{XMCD}(H)$, by measuring the hysteresis loop at the Co $K$ edge at $T=150 \mathrm{~K} . H_{\mathrm{C} \perp}$ is reduced from $3.6 \mathrm{kOe}$ down to $0.39 \mathrm{kOe}$, for $\mathrm{XMCD}(H)$ curves measured at 12 and $150 \mathrm{~K}$, respectively. This behavior is consistent with that observed by SQUID and AHE measurements (see Fig. 14).

\section{DISCUSSION}

The formation of a $\mathrm{Co}_{x} \mathrm{Pt}_{1-x}$ alloy in our Co-Pt granular films has been proven by XANES and EXAFS measurements at the Co $K$ and $\mathrm{Pt} L_{3}$ edges. It results from the interdiffusion of Co atoms from the previously formed Co clusters towards the Pt matrix, as observed in other Co-Pt systems produced by sputtering [11] and LECBD [61] at room temperature. The composition of the $\mathrm{Co}_{x} \mathrm{Pt}_{1-x}$ alloy present in all Co-Pt granular samples seems to be close to $\mathrm{Co}_{0.5} \mathrm{Pt}_{0.5}$, according to the EXAFS results. In fact, the morphology of our Co-Pt granular samples may be visualized as that of a $\mathrm{Co}_{0.5} \mathrm{Pt}_{0.5}$ alloy surrounding the Co rich core, embedded in metallic Pt, in view of the particulate morphology of the Co-Pt layers observed by TEM and the structure described by EXAFS measurements. Recent calculations of core-shell morphologies in CoPt nanoparticles [62,63] have demonstrated that the Co core- $\mathrm{Co}_{0.5} \mathrm{Pt}_{0.5}$ alloy shell, with some segregation of $\mathrm{Pt}$ atoms 
to the particle surface, is one of the most energetically stable configurations of NPs with diameter close to $3 \mathrm{~nm}$ (clusters of $\sim 1000$ atoms).

The presence of this alloy in our Co-Pt granular films gives rise to a quite different magnetic behavior than the blocked superparamagnetic one observed in Co- $M$ NPs with $M=\mathrm{Cu}, \mathrm{Ag}, \mathrm{Au}$, and $\mathrm{W}[6,7]$. The different magnetometry techniques employed to study the Co-Pt granular films reveal the presence of PMA in all the samples in the series. Similar PMA behavior has been observed in several Co-Pt multilayer systems [21-24], $\mathrm{Co}_{x} \mathrm{Pt}_{1-x}$ alloy films [11,25-27], and nanopatterned Co-Pt systems [28], and is correlated to the structure of the alloy present in the sample. For instance, CoPt alloys with composition close to $\mathrm{Co}_{0.5} \mathrm{Pt}_{0.5}$ may have a disordered fcc structure or a chemically ordered tetragonal structure $L 1_{0}$. Both phases are ferromagnetic and the $c$ axis of the $L 1_{0}$ is an easy direction of magnetization. Co-Pt films grown with this $L 1_{0}$ phase show PMA [27]. Therefore one may identify the PMA appearing on our Co-Pt samples as due to the formation of $\mathrm{Co}_{0.5} \mathrm{Pt}_{0.5}$ alloy with short range ordering in the $L 1_{0}$ structure. The XMCD data also point towards the same interpretation, since it allowed to demonstrate that the origin of the PMA in our Co-Pt films is at the anisotropy of the orbital moment, as it has been observed on different $\mathrm{Co}-\mathrm{Pt}$ systems reported in the literature [27,32]. The polarization of the Pt atoms (identified from XMCD measurements) as consequence of the Co $3 d-\mathrm{Pt} 5 d$ hybridization in the $\mathrm{Co}-\mathrm{Pt}$ films also plays an important role in this anisotropic properties. It results from the strong Pt spin-orbit coupling $\left(\xi_{5 d}=0.66\right.$ eV) [64] that favors a large perpendicular magnetocrystalline anisotropy in Co-Pt systems, as observed in $L 1_{0} \mathrm{Co}_{0.5} \mathrm{Pt}_{0.5}$ alloy films [27].

The anisotropy constant of Co-Pt alloy films at $T=$ $5 \mathrm{~K}$ with such an ordered structure is on the order of $K_{\text {eff }}=10^{7} \mathrm{erg} / \mathrm{cm}^{3}$ [27,32]. The $K_{\text {eff }}$ values estimated for our Co-Pt granular films (see Sec. V) are found to be of this same order $\left(1-4.8 \times 10^{7} \mathrm{erg} / \mathrm{cm}^{3}\right)$. These values are in contrast to lower $K_{\text {eff }}$ obtained for Co-Pt clusters prepared by LECBD with highly-ordered $L 1_{0}$ structure, for which $K_{\text {eff }}=0.19 \times 10^{7} \mathrm{erg} / \mathrm{cm}^{3}$ for as-prepared and $K_{\text {eff }}=$ $0.38 \times 10^{7} \mathrm{erg} / \mathrm{cm}^{3}$ for annealed particles [10]. However, the estimation of the anisotropy values in our granular films make use of several approximations, and can only be compared qualitatively to that of the $L 1_{0}$ Co-Pt alloy thin film and other values reported in the literature.

According to the phase diagram showed in Fig. 9, the temperature dependence of the magnetic behavior of the granular Co-Pt films can be divided into three magnetic regions, namely:

(i) $0<T<T_{1}$ : this region has the characteristic behavior of ferromagnetic granular layers, with interlayer ferromagnetic coupling. This statement is supported by the square shape of the hysteresis curves and the high $H_{\mathrm{C} \perp}$ values obtained at $T=5 \mathrm{~K}$ when the field is applied perpendicular to the substrate (see Fig. 19). These squared curves also demonstrate that the Co-Pt grains in the samples are strongly magnetically coupled. In all cases studied, the samples show strong PMA anisotropy (see Fig. 10). In fact, the inversion of the film magnetization direction upon reduction and switching sign of the applied field perpendicular to the sample plane takes place in steps [see Fig. 13(b)]. This is a proof that each layer acts as a ferromagnetic system coupled to the adjacent ones by a weaker interaction. The difference between the FC and ZFC curves is ascribed to the pinning of the ferromagnetic domain walls. The most probable intrafilm interaction between the grains is of long range RKKY type through the connecting Pt metal. We support this statement by the observation of magnetically polarized Pt by means of XMCD measurements at the $\mathrm{Pt} L_{2,3}$ edges. Besides, in the hysteresis curves there is some evidence of a soft magnetic Co core [see feature A in Fig. 10(a)]. As the temperature increases approaching $T_{1}$, the intra film graingrain interaction weakens and the coercive field decreases, being zero at a temperature of $T_{H c} \approx 200 \mathrm{~K}$ in most samples. As the bias field increases, this hard ferromagnetic phase tends to be quenched and actually disappears at the $H_{\mathrm{dc}} \geqslant H_{\mathrm{C} \perp}$, as $T$ tends to zero (see Fig. 7), i.e., when the applied field orients all ferromagnetic domains parallel to its direction and the magnetization is saturated. This common behavior to all samples suggests that the magnetic hard component is the same along the series.

(ii) $T_{1}<T<T_{f}$ : this state is very distinctive in having no coercivity, in contrast to region (i). However, the hysteresis curve measured with the field in the perpendicular configuration, $M_{\perp}(H)$, is also very square, while the one measured with the parallel configuration, $M_{\|}(H)$ has a rounded shape [see Fig. 10(b)]. Thus it behaves as a soft ferromagnet with PMA. The absence of coercivity can be related to the weakening of the intergrain interactions so that the grains are predominantly magnetically coupled within the layer with short-range correlations. Since the coercive field becomes lower than the dc applied field, the ZFC magnetization increases towards the FC curve, as in amorphous asperomagnets [65]. This intermediate behavior is sustained for increasing temperature till a maximum in the $M_{\perp}(H)$ curve indicates the freezing temperature $T_{f}$, where the FC-ZFC curves merge. For increasing bias field, the intermediate phase range reduces since $T_{f}$ decreases till actual disappearance for an applied field of about $20 \mathrm{kOe}$. This phase can be described as an asperomagneticlike phase in which the spins are oriented in fixed directions, with most nearest-neighbor spins parallel or nearly parallel, so that the spin directions are distributed in one hemisphere below a $T_{f}$ temperature. As the bias field increases, region (ii) tends to disappear by polarization of all moments along the applied field direction.

(iii) $T>T_{f}$ : intergrain interactions in this region become very weak with respect to the thermal energy and the behavior becomes that of an isotropic superparamagnet as evidenced by the two identical $M(H)$ curves, irrespective of field orientation [see Fig. 10(c)]. They are fitted very well for the prediction of $M(H)$ for a collection of independent particles subject to an external field.

Evidently, the intergranular Pt plays an important role in establishing short and long range correlations. At a coverage of $t_{\mathrm{Pt}}=0.6 \mathrm{~nm}$, the interaction is ferromagnetic due to the percolated metallic Pt matrix that supports the CoPt alloyed grains. At larger cappings, and specially at $t_{\mathrm{Pt}}=6.0 \mathrm{~nm}$ the excess of Pt filling all intergranular spaces has the effect of decoupling the CoPt correlated grains. Interestingly, the value of $T_{f}$ decreases and the effective anisotropy $K_{\text {eff }}$ decreases with increasing nominal Pt thickness (see Table III). In an 
interesting paper on the intergranular interactions caused by the interspacial metallic $\mathrm{Pt}$ in a monolayer of Co-Pt granular film, similar features, such as the existence of an intermediate region at $T_{1}<T<T_{f}$ and the weakening of the interaction when an excess of $\mathrm{Pt}$ is present, are found for $t_{\mathrm{Pt}}$ comparable to our case [66]. Therefore, and similarly to the Co-Pt granular films described in Ref. [66], the coupling/decoupling between our Co-Pt grains can be tailored by variation of the amount of $\operatorname{Pt}\left(t_{\mathrm{Pt}}\right)$ in the samples.

Finally, we can compare the XMCD results in our Co-Pt granular films with those of recent calculations of the spin magnetic moments of $\mathrm{Co}$ and $\mathrm{Pt}$ atoms in Co core- $\mathrm{Co}_{0.5} \mathrm{Pt}_{0.5}$ alloy shell clusters of similar sizes [62,63]. For the latter, values of $m_{\mathrm{S}}^{\mathrm{Co}}=1.85-1.96 \mu_{B} / \mathrm{Co}$ atom and $m_{\mathrm{S}}^{\mathrm{Pt}}=0.26-0.42 \mu_{B} / \mathrm{Pt}$ atom are obtained $[62,63]$. We have estimated the spin moment in Co in our Co-Pt granular films to range between $m_{\mathrm{S}}^{\text {Co }}=$ 1.27-1.34 $\mu_{B} / \mathrm{Co}$, assuming the number of unoccupied states in the $3 d$ band for Co to be between the $n_{h}=2.49$ holes for metallic Co [54] and $n_{h}=2.628$ for $\mathrm{Co}_{0.5} \mathrm{Pt}_{0.5} L 1_{0}$ alloy films [27]; thus, the $m_{\mathrm{S}}^{\mathrm{Co}}$ in our Co-Pt system is lower than that calculated for clusters of similar morphology. In the case of $m_{\mathrm{S}}^{\mathrm{Pt}}$, the deviation of the $m_{\mathrm{S}}^{\mathrm{Pt}}=0.14(1) \mu_{B}$ value for our Co-Pt samples from that calculated in Refs. [62,63] may be related to the difference between the actual amount of Pt in the samples that is polarized by Co and contributes to the XMCD signal, as described in Sec. VII. Alternatively, for the sake of comparison, we may estimate the total magnetic moment induced in the Pt atoms $m_{\mathrm{T}}^{\mathrm{Pt}}$ in our Co-Pt samples from the difference between the total magnetic moment contributing to the saturation magnetization measured in the SQUID and that of the total magnetic moment in Co atoms obtained from XMCD. Following this procedure, we obtain values of $m_{\mathrm{T}}^{\mathrm{Pt}}=$ $0.2-0.25 \mu_{B} / \mathrm{Pt}$ atom which are closer to the $m_{\mathrm{S}}^{\mathrm{Pt}}=0.26-0.42$ $\mu_{B} / \mathrm{Pt}$ atom values from the calculations.

\section{CONCLUDING REMARKS}

The overall picture describing the Co-Pt granular films obtained by room temperature sequential sputtering deposition is that of a Co-core with a Co-Pt alloy shell particles, embedded in the nonreacted Pt. The alloy between the Co and Pt results from interdiffusion of the atoms, similarly to what is observed in other Co-Pt systems [11]. The short-range order within the grains creates the anisotropy in the $\mathrm{Co}_{0.5} \mathrm{Pt}_{0.5}$ alloy, giving rise to the PMA, as consequence of the anisotropy of the orbital moment of the Co atoms in this alloy. The PMA in our Co-Pt films is on the order of $10^{7} \mathrm{erg} / \mathrm{cm}^{3}$, comparable to that of highly-ordered CoPt $L 1_{0}$ alloy films [27]. The polarization of the Pt atoms is observed by XMCD measurements and has been demonstrated to have an important role in the magnetic behavior of the Co-Pt granular films, not only in their anisotropic properties, but also in the magnetic coupling between the grains. The collective magnetic behavior of the Co-Pt granular films is temperature dependent, namely, below $T_{1}$ the granular sample is ferromagnetic with a high coercive field; for $T_{1}<T<T_{f}$ the granular film behaves as an amorphous asperomagnet, with a coupling between the grains mediated by the $\mathrm{Pt}$; and above $T_{f}$ the sample has a superparamagnetic behavior. The coupling/decoupling between the grains in our Co-Pt granular films can be tailored by variation of the amount of $\mathrm{Pt}$ in the samples.

The granular CoPt multilayers deposited on $\mathrm{Al}_{2} \mathrm{O}_{3}$ show strong PMA, with high coercivity at $5 \mathrm{~K}$. The magnetocrystalline anisotropy of the $\mathrm{Co}_{0.5} \mathrm{Pt}_{0.5}$ alloy in the $L 1_{0}$ phase, that forms during the capping process, is the cause of this behavior. A rich magnetic phase diagram evolves from the intra- and interlayer interactions.

\section{ACKNOWLEDGMENTS}

The financial support of the Spanish Ministerio de Economía y Competitividad (MINECO) MAT2011-23791 and Aragonese DGA-IMANA E34 (cofunded by Fondo Social Europeo) projects is acknowledged. Authors also acknowledge the Servicio General de Apoyo a la Investigación-SAI, Universidad de Zaragoza. A. I. F. acknowledges a Spanish Consejo Superior de Investigaciones Científicas (CSIC) JAE2008-Predoc grant. European Synchrotron Radiation Facility (ESRF) beamtime corresponds to HE2238, HE2541, HE2952, and HE3136 experiments.
[1] J. Bansmann, S. H. Baker, C. Binns, J. A. Blackman, J. P. Bucher, J. D. Dávila, V. Dupuis, L. Favre, D. Kechrako, A. Kleibert, K. H. Meiwes-Broer, G. M. Pastor, A. Perez, O. Toulemonde, K. N. Trohidou, J. Tuaillon, and Y. Xie, Surf. Sci. Rep. 56, 189 (2005).

[2] F. Luis, F. Petroff, J. M. Torres, L. M. García, J. Bartolomé, J. Carrey, and A. Vaurès, Phys. Rev. Lett. 88, 217205 (2002).

[3] J. Bartolomé, F. Luis, F. Petroff, F. Bartolomé, L. M. García, V. Cross, and H. Jaffres, Phys. Met. Metallogr. 99, S8 (2005).

[4] J. Bartolomé, F. Bartolomé, L. M. García, F. Luis, F. Petroff, V. Cros, H. Jaffrès, and A. Vaurès, in Smart Materials for Ranging Systems, edited by J. Franse, V. Eremenko, and V. Sirenko, Nato Science Series II Vol. 226 (Springer, Netherlands, 2006), pp. 1-25.

[5] F. Luis, F. Bartolomé, F. Petroff, J. Bartolomé, L. M. García, C. Deranlot, H. Jaffrès, M. J. Martínez, P. Bencok, F. Wilhelm, A. Rogalev, and N. Brookes, Europhys. Lett. 76, 142 (2006).
[6] J. Bartolomé, L. M. García, F. Bartolomé, F. Luis, R. LópezRuiz, F. Petroff, C. Deranlot, F. Wilhelm, A. Rogalev, P. Bencok, N. B. Brookes, L. Ruiz, and J. M. González-Calbet, Phys. Rev. B 77, 184420 (2008).

[7] A. I. Figueroa, J. Bartolomé, L. M. García, F. Bartolomé, C. Magén, A. Ibarra, L. Ruiz, J. M. González-Calbet, F. Petroff, C. Deranlot, S. Pascarelli, P. Bencok, N. B. Brookes, F. Wilhelm, and A. Rogalev, Phys. Rev. B 84, 184423 (2011).

[8] M. T. Johnson, P. J. H. Bloemen, F. J. A. den Broeder, and J. J. de Vries, Rep. Prog. Phys. 59, 1409 (1996).

[9] S. Rohart, C. Raufast, L. Favre, E. Bernstein, E. Bonet, and V. Dupuis, Phys. Rev. B 74, 104408 (2006).

[10] F. Tournus, A. Tamion, N. Blanc, A. Hannour, L. Bardotti, B. Prével, P. Ohresser, E. Bonet, T. Epicier, and V. Dupuis, Phys. Rev. B 77, 144411 (2008).

[11] K. K. M. Pandey, J. S. Chen, T. Liu, C. J. Sun, and G. M. Chow, J. Phys. D: Appl. Phys. 42, 185007 (2009). 
[12] J. Bartolomé, A. I. Figueroa, L. M. García, F. Bartolomé, L. Ruiz, J. M. González-Calbet, F. Petroff, C. Deranlot, F. Wilhelm, A. Rogalev, and N. Brookes, Low Temp. Phys. 38, 835 (2012).

[13] K. H. J. Buschow, P. van Engen, and R. Jongebreur, J. Magn. Magn. Mater 38, 1 (1983).

[14] M. Naoe, H. Kazama, Y. Hoshi, and S. Yamanaka, J. Appl. Phys. 53, 7846 (1982).

[15] K. H. J. Buschow, J. Appl. Phys. 54, 2578 (1983).

[16] J. Dubowik, Y. V. Kudryavtsev, and R. Gontarz, Acta Phys. Pol. A 76, 331 (1989).

[17] M. A. M. Ibrahim, S. S. A. E. Rehim, and S. O. Moussa, J. Appl. Electochem. 33, 627 (2003).

[18] J. Arbiol, F. Peiro, A. Cornet, C. Clavero, A. Cebollada, G. Armelles, and Y. Huttel, Appl. Phys. Lett. 86, 032510 (2005).

[19] A. Ebbing, O. Hellwig, L. Agudo, G. Eggeler, and O. Petracic, Phys. Rev. B 84, 012405 (2011).

[20] G. Hadjipanayis and P. Gaunt, J. Appl. Phys. 50, 2358 (1979).

[21] P. F. Carcia, J. Appl. Phys. 63, 5066 (1988).

[22] S. Hashimoto, Y. Ochiai, and K. Aso, J. Appl. Phys. 66, 4909 (1989).

[23] C. H. Lee, R. F. C. Farrow, C. J. Lin, E. E. Marinero, and C. J. Chien, Phys. Rev. B 42, 11384 (1990).

[24] N. Nakajima, T. Koide, T. Shidara, H. Miyauchi, H. Fukutani, A. Fujimori, K. Iio, T. Katayama, M. Nývlt, and Y. Suzuki, Phys. Rev. Lett. 81, 5229 (1998).

[25] M. Maret, M. C. Cadeville, W. Staiger, E. Beaurepaire, R. Poinsot, and A. Herr, Thin Solid Films 275, 224 (1996).

[26] W. Grange, M. Maret, J.-P. Kappler, J. Vogel, A. Fontaine, F. Petroff, G. Krill, A. Rogalev, J. Goulon, M. Finazzi, and N. B. Brookes, Phys. Rev. B 58, 6298 (1998).

[27] W. Grange, I. Galanakis, M. Alouani, M. Maret, J.-P. Kappler, and A. Rogalev, Phys. Rev. B 62, 1157 (2000).

[28] D. Makarov, E. Bermúdez-Ureña, O. G. Schmidt, F. Liscio, M. Maret, C. Brombacher, S. Schulze, M. Hietschold, and M. Albrecht, Appl. Phys. Lett. 93, 153112 (2008).

[29] N. Nishimura, T. Hirai, A. Koganei, T. Ikeda, K. Okano, Y. Sekiguchi, and Y. Osada, J. Appl. Phys. 91, 5246 (2002).

[30] S. Ikeda, K. Miura, H. Yamamoto, K. Mizunuma, H. D. Gan, M. Endo, S. Kanai, J. Hayakawa, F. Matsukura, and H. Ohno, Nat. Mater. 9, 721 (2010).

[31] Q. F. Xiao, E. Bruck, Z. D. Zhang, F. R. de Boer, and K. H. J. Buschow, J. Alloys. Comp. 364, 64 (2004).

[32] M. Sakamaki, K. Amemiya, M. O. Liedke, J. Fassbender, P. Mazalski, I. Sveklo, and A. Maziewski, Phys. Rev. B 86, 024418 (2012).

[33] D. Babonneau, F. Petroff, J. L. Maurice, F. Fettar, A. Vaures, and A. Naudons, Appl. Phys. Lett. 76, 2892 (2000).

[34] F. Luis, J. M. Torres, L. M. García, J. Bartolomé, J. Stankiewicz, F. Petroff, F. Fettar, J. L. Maurice, and A. Vaures, Phys. Rev. B 65, 094409 (2002).

[35] J. Briático, J.-L. Maurice, J. Carrey, D. Imhoff, F. Petroff, and A. Vaurès, Eur. Phys. J. D 9, 517 (1999).

[36] D. Babonneau, D. Lantiat, S. Camelio, J. Toudert, L. Simonot, F. Pailloux, M.-F. Denanot, and T. Girardeau, Eur. Phys. J. Appl. Phys. 44, 3 (2008).

[37] B. Ravel and M. Newville, J. Synchrotron Rad. 12, 537 (2005).

[38] http://icsd.iqfr.csic.es/icsd/

[39] B. Ravel, J. Synchrotron Rad. 8, 314 (2001).
[40] Y. S. Lee, J. Y. Rhee, C. N. Whang, and Y. P. Lee, Phys. Rev. B 68, 235111 (2003).

[41] N. Blanc, L. E. Díaz-Sánchez, A. Y. Ramos, F. Tournus, H. C. N. Tolentino, M. De Santis, O. Proux, A. Tamion, J. TuaillonCombes, L. Bardotti, O. Boisron, G. M. Pastor, and V. Dupuis, Phys. Rev. B 87, 155412 (2013).

[42] J.-I. Park, M. G. Kim, Y. wook Jun, J. S. Lee, W. ram Lee, and J. Cheon, J. Am. Chem. Soc. 126, 9072 (2004).

[43] S. Mukerjee, S. Srinivasan, M. P. Soriaga, and J. McBreen, J. Electrochem. Soc. 142, 1409 (1995).

[44] M. Négrier, J. Tuaillon-Combes, V. Dupuis, and A. P. P. Mélinon, Philos. Mag. A 81, 2855 (2001).

[45] J. W. Knepper and F. Y. Yang, Phys. Rev. B 71, 224403 (2005).

[46] A. Gerber, A. Milner, M. Karpovsky, B. Lemke, H.-U. Habermeier, J. Tuaillon-Combes, M. Négrier, O. Boisron, P. Mélinon, and A. Perez, J. Magn. Magn. Mater. 242-245, 90 (2002).

[47] A. Gerber, A. Milner, J. Tuaillon-Combes, M. Négrier, O. Boisron, P. Mélinon, and A. Perez, J. Magn. Magn. Mater. 241, 340 (2002).

[48] S. Pizzini, A. Fontaine, C. Giorgetti, E. Dartyge, J.-F. Bobo, M. Piecuch, and F. Baudelet, Phys. Rev. Lett. 74, 1470 (1995).

[49] A. Maziewski, P. Mazalski, Z. Kurant, M. O. Liedke, J. McCord, J. Fassbender, J. Ferré, A. Mougin, A. Wawro, L. T. Baczewski, A. Rogalev, F. Wilhelm, and T. Gemming, Phys. Rev. B 85, 054427 (2012).

[50] Y. Joly, Phys. Rev. B 63, 125120 (2001).

[51] O. Bunău and Y. Joly, J. Phys.: Condens. Matter 21, 345501 (2009).

[52] B. T. Thole, P. Carra, F. Sette, and G. van der Laan, Phys. Rev. Lett. 68, 1943 (1992).

[53] P. Carra, B. T. Thole, M. Altarelli, and X. Wang, Phys. Rev. Lett. 70, 694 (1993)

[54] M. G. Samant, J. Stohr, S. S. P. Parkin, G. A. Held, B. D. Hermsmeier, F. Herman, M. van Schilfgaarde, L.-C. Duda, D. C. Mancini, N. Wassdahl, and R. Nakajima, Phys. Rev. Lett. 72, 1112 (1994).

[55] S. Stepanow, A. Mugarza, G. Ceballos, P. Moras, J. C. Cezar, C. Carbone, and P. Gambardella, Phys. Rev. B 82, 014405 (2010).

[56] J. Stöhr and H. König, Phys. Rev. Lett. 75, 3748 (1995).

[57] P. Imperia, P. Andreazza, D. Schnitz, J. Penuelas, and C. Andreazza-Vignole, J. Magn. Magn. Mater 310, 2417 (2007).

[58] P. Imperia, L. Glaser, M. Martins, P. Andreazza, J. Penuelas, V. Alessandrovic, H. Weller, C. Andreazza-Vignolle, and W. Wurth, Phys. Stat. Sol. (a) 205, 1047 (2008).

[59] A. I. Figueroa, F. Bartolomé, J. Bartolomé, L. M. García, F. Petroff, C. Deranlot, F. Wilhelm, and A. Rogalev, Phys. Rev. B 86, 064428 (2012).

[60] E. C. Stoner, Proc. R. Soc. A 165, 372 (1938).

[61] M. Jamet, M. Négrier, V. Dupuis, J. Tuaillon-Combes, P. Mélinon, A. Pérez, W. Wernsdorfer, B. Barbara, and B. Baguenard, J. Magn. Magn. Mater. 237, 293 (2001).

[62] M. E. Gruner, J. Phys.: Conf. Ser. 200, 072039 (2010).

[63] M. E. Gruner, J. Phys. D: Appl. Phys. 43, 474008 (2010).

[64] A. R. Mackintosh and O. K. Andersen, in Electrons at the Fermi Surface, edited by M. Springford (Cambridge University Press, New York, 1980), Chap. 5, pp. 149-224.

[65] J. M. D. Coey, Can. J. Phys. 65, 1210 (1987).

[66] A. Ludwig, L. Agudo, G. Eggeler, A. Ludwig, A. D. Wieck, and O. Petracic, J. Appl. Phys. 113, 043917 (2013). 